\title{
An Application of (3+1)-Dimensional Time-Space Fractional ZK Model to Analyze the Complex Dust Acoustic Waves
}

\author{
Lei Fu and Hongwei Yang (D) \\ Shandong University of Science and Technology, Qingdao, Shandong 266590, China \\ Correspondence should be addressed to Hongwei Yang; hwyang1979@163.com
}

Received 25 April 2019; Revised 26 June 2019; Accepted 22 July 2019; Published 5 August 2019

Guest Editor: Robert Hakl

Copyright ( 2019 Lei Fu and Hongwei Yang. This is an open access article distributed under the Creative Commons Attribution License, which permits unrestricted use, distribution, and reproduction in any medium, provided the original work is properly cited.

\begin{abstract}
Dust plasma is a new field of physics which has developed rapidly in recent decades. The study of dust plasma has received much attention due to its importance in the environment of space and the Earth. Dust acoustic waves are generated because of the inertia of dust mass while the restoring force is provided by the thermal pressure of electrons and ions. Since dust acoustic waves were first reported theoretically in unmagnetized dust plasma by Rao et al., they have become a research hot spot. In this paper, the excitation of dust acoustic waves by a gravity field in a dust plasma is analyzed. According to the control equations of dust plasma motion and employing multiscale analysis and perturbation method, we have obtained a (3+1)-dimensional ZK model. Because of the space property of dust plasma, (3+1)-dimensional ZK equation is more suitable than $\mathrm{KdV}$ equation and (2+1)-dimensional ZK equation to describe the real dust acoustic waves. Then, the (3+1)-dimensional time-space fractional ZK (TSF-ZK) equation describing the fractal process of nonlinear dust acoustic waves is given for the first time. To further explore how dust acoustic waves change energy as they travel, we discuss the conservation laws of the new model. Moreover, we study the exact solution of (3+1)-dimensional TSFZK equation by using extended Kudryashov method. Finally, based on the exact solution, we further investigate the effect of the parameter $k$, the charge properties of dust particle $Z_{d 0}$, the fractional order values $\alpha, \beta, \gamma$, and $\theta$, the temperature $T_{d}$, the gravity $g$, and the collision frequency $\beta_{0}$ and $\beta_{1}$ on the properties of dust acoustic waves by a gravity field in dust plasma.
\end{abstract}

\section{Introduction}

Plasma is a macroscopic system composed of a large number of charged particles, and it is the fourth state in which matter exists. The characteristics of motion of plasma are more complex than those of other substances. In many cases, the production and maintenance of plasma are difficult. Therefore, plasma physics is a branch with research value, which is closely related to the generation of new technology. In recent years, plasma physics has become an important basis for human understanding of the universe and an important guarantee for understanding and controlling the changes of the Earth's environment. It is hoped that mankind will finally solve the energy problem in the future. As a result, plasmas are getting more attention.

In recent years, the study of nonlinear structures in various kinds of plasmas has been the emphasis of the researchers $[1,2]$. Dust plasma is a new field of physics research which has developed rapidly in recent decades. In addition to electrons, ions, and neutral gases, dust plasma also contains dust particles of any shape and size ranging from tens of nm to a few microns. Charged dust grains exist widely in space, plasma equipment in laboratory as well as plasma processing. It is widely believed that dust plasma plays a very important role in the formation of galaxies, such as planetary rings, cometary surroundings, interstellar clouds, and the Earth's ionosphere. Dust plasma is also important in the laboratory. And the main reason is that dust plasma mode is formed due to the existence of mixtures, which greatly affects the state or behavior of plasma. The dust acoustic waves are generated due to the inertia of dust mass while the restoring force is provided by the thermal pressure of electrons and ions. Low frequency dust acoustic waves were first predicted by Rao [3] in a dust plasma in 1990. Later, in 1992, Shukla-Silin [4] obtained the high frequency dust ionacoustic waves. Merlino [5] has experimentally confirmed the 
existence of dust acoustic waves and dust ion-acoustic waves in 1998. Since then, more and more attention has been paid to the study of dust acoustic waves.

At the beginning of the investigations, the $(1+1)$ dimensional model was used to study the dust acoustic waves. Using the reductive perturbation method, a $\mathrm{KdV}$ equation has been derived by Bharuthram [6] to study the large amplitude ion-acoustic solitons in a dust plasma, and Duan [7] got a KdV equation to describe the effect of dust size distribution for two ion temperature dusty plasma. Later, Kadomstev and Petviashvili [8] made an attempt to describe the solitons in $(2+1)$-dimensional systems by applying Kadomstev-Petviashvili (KP) equation. Singh and Honzawa [9] studied the effects of ion temperature and relativistic factor on the width and amplitude of ion-acoustic solitons in an unmagnetized two-dimensional weakly relativistic collisionless plasma with finite ion temperature by using the KP equation. Lin [10] derived the KP equation which is considered as two-dimensional extension of $\mathrm{KdV}$ equation to study dust acoustic waves in hot dusty plasma. Employing multiscale analysis and perturbation method, a $\mathrm{KP}$ equation was obtained for the stability of dust acoustic waves for host dust plasma by Duan [11]. Gill [12] proved the existence of compressive and rarefactive dust acoustic solitons under the solution of KP equation in two-dimensional dusty plasma with two-temperature ions. However, in the real environment, dust plasma moves in space, so we must study the higher dimensions of dust acoustic waves. Thus, in this paper, we will be working on the $(3+1)$-dimensional model.

For a long time, people have been committed to the study of integral calculus. Therefore, researchers conduct scientific research based on integer order models [13-18]. However, with the continuous development of calculus theory, the appearance and development of fractional order have become a major trend of integral science [19-21]. As we know, Leibniz was the first to study the theory of fractional derivatives. So far, the development of fractional derivatives has been very mature, and fractional calculus and the corresponding fractional partial differential equations have attracted wide attention in many subjects [22-28]. Compared with integer order models, fractional models can better explain nonlinear physical processes and propagation characteristics in real environment [29-33]. However, fractional models were rarely used to study the dust plasma in the past. Therefore, it is of great research value to construct the $(3+1)$-dimensional TSF$\mathrm{ZK}$ equation to discuss the influence of fractional order for the dust acoustic waves in gravity field for dust plasma.

The solution of the nonlinear partial differential equation is an important research topic in nonlinear physical phenomena. Similarly, the solutions of fractional differential equations have attracted the attention of researchers in many fields. Thus, many methods have been proposed one after another, such as the first integral method [34, 35], (G'/G)expansion method [36, 37], the Hirota method [38, 39], the trial function method [40], the subequation method [41], and others [42-46]. In this paper, using extended Kudryashov method [47], the exact solution of (3+1)-dimensional TSF-ZK equation is obtained.
The rest of the paper is organized as follows. In Section 2, based on the control equations of motion, a $(3+1)$ dimensional integer order ZK equation is derived by employing multiscale analysis and perturbation method [48, 49]. Applying the semi-inverse method and the fractional variational principle [50], the integer order ZK equation is transformed into a time-space fractional ZK (TSF-ZK) equation. Next, we study the exact solution of $(3+1)$-dimensional TSF$\mathrm{ZK}$ equation by using extended Kudryashov method. Then, the conservation law of $(3+1)$-dimensional TSF-ZK equation is got by applying Lie symmetry analysis method. In the end, on the basis of the exact solution of $(3+1)$-dimensional TSF-ZK equation, we further investigate the property of dust acoustic waves. The influence of the parameter $k$, the charge properties of dust particle $Z_{d 0}$, fractional order values $\alpha, \beta$, $\gamma, \theta$, temperature $T_{d}$, gravity $g$, and collision frequency $\beta_{0}, \beta_{1}$ on the properties of dust acoustic waves by a gravity field in dust plasma are studied.

\section{Derivation of the $(3+1)$-Dimensional ZK Equation}

For low frequency dust acoustic wave, the control equations are composed of mass conservation equation, momentum conservation equation, and Poisson equation [51, 52] following

$$
\begin{aligned}
& \frac{\partial n_{d}}{\partial t}+\nabla\left(n_{d} \overrightarrow{u_{d}}\right)=0 \\
& n_{d} m_{d} \frac{\partial \overrightarrow{u_{d}}}{\partial t}+n_{d} m_{d} \overrightarrow{u_{d}} \cdot \nabla \overrightarrow{u_{d}}+T_{d} \nabla \overrightarrow{u_{d}}-n_{d} e Z_{d} \nabla \phi \\
& \quad=-\beta^{\prime} n_{d} m_{d} \overrightarrow{u_{d}}+n_{d} m_{d} \vec{g} \\
& \Delta \phi=4 \pi e\left(Z_{d} n_{d}+n_{e}-n_{i}\right)
\end{aligned}
$$

where $\Delta=\nabla^{2}=\partial^{2} / \partial x^{2}+\partial^{2} / \partial y^{2}+\partial^{2} / \partial z^{2}$ is three-dimensional Laplace operator, $\overrightarrow{u_{d}}$ is the velocity of dust particles, $\phi$ is the electrostatic potential, $n_{d}, n_{e}$, and $n_{i}$ are the number densities of the dust particles, electrons, and ions in the dusty plasma, respectively, $T_{d}$ is the temperature of dust particles, $Z_{d}$ is the basic unit electric quantity of dust particles, and its positive and negative represent the electrical properties of dust particles. $\overrightarrow{u_{d}}$ is expressed by $\overrightarrow{u_{d}}=u_{d} \vec{i}+v_{d} \vec{j}+w_{d} \vec{k}$, where $\vec{i}$, $\vec{j}$, and $\vec{k}$ are the unit vectors in the $x, y$, and $z$ directions, respectively. $n_{e}=\nu e^{s \delta \phi}$ and $n_{i}=\mu e^{-s \phi}$, where $s=1 /(\mu+\nu \delta)$, $\delta=T_{e} / T_{i}, T_{e f f}=T_{e} T_{i} /\left(\mu T_{e}+\nu T_{i}\right)$ is the effective temperature, where $T_{e}$ and $T_{i}$ are the temperatures of electrons and ions, respectively, $\mu=n_{i 0} / n_{d 0} Z_{d 0}$, and $\nu=n_{i 0} / n_{d 0} Z_{d 0}$. In a state of complete constant temperature, the ions and electrons will obey the Boltzmann condition. The equilibrium state satisfies the neutral condition $n_{i 0}=Z_{d 0} n_{d 0}+n_{e 0}$, where $n_{d 0}$, $n_{i 0}, n_{e 0}$, and $Z_{d 0}$ are the unperturbed number densities of dust particles, ions, electrons, and the numbers of electrons residing on the dust particles, respectively. $n_{d}$ is normalized by $n_{d 0}$, and $Z_{d}$ is normalized by $Z_{d 0}$. The space coordinates $(x, y, z)$, time $t$, velocity $\left(u_{d}, v_{d}, w_{d}\right)$, electrostatic potential $\phi$, collision frequency $\beta^{\prime}$, and gravity $g$ are normalized by 
Debye length $\lambda_{D d}=\left(T_{e f f} / 4 \pi Z_{d 0} n_{d 0} e^{2}\right)^{1 / 2}$, the characteristic dust period $\omega_{p d}^{-1}=\left(m_{d} / 4 \pi Z_{d 0} n_{d 0} e^{2}\right)^{1 / 2}$, the dust acoustic speed $C_{d}=\left(Z_{d 0} T_{e f f} / m_{d}\right)^{1 / 2}$, and $T_{e f f} / e$, respectively, and the collision frequency $\beta^{\prime}$ refers to the number of collisions between one plasma and another over a period of time. The detailed description is given in [45].

We assume that the charge of dust particles is constant; that is, $Z_{d}=Z_{d 0}, Z_{d 0}$ is constant. Then the dimensionless forms of the control equations are as follows:

$$
\begin{aligned}
& \frac{\partial n_{d}}{\partial t}+\frac{\partial}{\partial x}\left(n_{d} u_{d}\right)+\frac{\partial}{\partial y}\left(n_{d} v_{d}\right)+\frac{\partial}{\partial z}\left(n_{d} w_{d}\right)=0, \\
& \frac{\partial u_{d}}{\partial t}+u_{d} \frac{\partial u_{d}}{\partial x}+v_{d} \frac{\partial u_{d}}{\partial y}+w_{d} \frac{\partial u_{d}}{\partial z}+\frac{T_{d}}{Z_{d}} \frac{1}{n_{d}} \frac{\partial n_{d}}{\partial x}-\frac{\partial \phi}{\partial x} \\
& =-\beta_{x} u_{d}+g, \\
& \frac{\partial v_{d}}{\partial t}+u_{d} \frac{\partial v_{d}}{\partial x}+v_{d} \frac{\partial v_{d}}{\partial y}+w_{d} \frac{\partial v_{d}}{\partial z}+\frac{T_{d}}{Z_{d}} \frac{1}{n_{d}} \frac{\partial n_{d}}{\partial y}-\frac{\partial \phi}{\partial y} \\
& =-\beta_{y} v_{d}, \\
& \frac{\partial w_{d}}{\partial t}+u_{d} \frac{\partial w_{d}}{\partial x}+v_{d} \frac{\partial w_{d}}{\partial y}+w_{d} \frac{\partial w_{d}}{\partial z}+\frac{T_{d}}{Z_{d}} \frac{1}{n_{d}} \frac{\partial n_{d}}{\partial z}-\frac{\partial \phi}{\partial z} \\
& =-\beta_{z} w_{d}, \\
& \frac{\partial^{2} \phi}{\partial x^{2}}+\frac{\partial^{2} \phi}{\partial y^{2}}+\frac{\partial^{2} \phi}{\partial z^{2}}=n_{d}+n_{e}-n_{i} .
\end{aligned}
$$

We introduce the following slow space-time variables:

$$
\begin{aligned}
& \xi=\epsilon^{1 / 2}\left(x-v_{0} t\right), \\
& \eta=\epsilon^{1 / 2} y, \\
& \zeta=\epsilon^{1 / 2} z, \\
& \tau=\epsilon^{3 / 2} t .
\end{aligned}
$$

Then we have

$$
\begin{aligned}
& \frac{\partial}{\partial t}=\epsilon^{3 / 2} \frac{\partial}{\partial \tau}-\epsilon^{1 / 2} v_{0} \frac{\partial}{\partial \xi} \\
& \frac{\partial}{\partial x}=\epsilon^{1 / 2} \frac{\partial}{\partial \xi} \\
& \frac{\partial}{\partial y}=\epsilon^{1 / 2} \frac{\partial}{\partial \eta} \\
& \frac{\partial}{\partial z}=\epsilon^{1 / 2} \frac{\partial}{\partial \zeta}
\end{aligned}
$$

The dependent variables $n_{d}, u_{d}, v_{d}, w_{d}$, and $\phi$ are expanded as follows:

$$
\begin{aligned}
& n_{d}=1+\epsilon n_{d 1}+\epsilon^{2} n_{d 2}+\epsilon^{3} n_{d 3}+\cdots, \\
& u_{d}=u_{d 0}+\epsilon u_{d 1}+\epsilon^{2} u_{d 2}+\epsilon^{3} u_{d 3}+\cdots,
\end{aligned}
$$

$$
\begin{aligned}
v_{d} & =\epsilon^{3 / 2} v_{d 1}+\epsilon^{5 / 2} v_{d 2}+\epsilon^{7 / 2} v_{d 3}+\cdots, \\
w_{d} & =\epsilon^{3 / 2} w_{d 1}+\epsilon^{5 / 2} w_{d 2}+\epsilon^{7 / 2} w_{d 3}+\cdots, \\
\phi & =\epsilon \phi_{1}+\epsilon^{2} \phi_{2}+\epsilon^{3} \phi_{3}+\cdots, \\
\beta_{x} & =\beta_{0}+\epsilon \beta_{1}, \\
\beta_{y} & =\epsilon \beta_{1}, \\
\beta_{z} & =\epsilon \beta_{1} .
\end{aligned}
$$

Substituting (4) and (5) into (3), the following equations can be got:

$$
\begin{aligned}
& \left(\epsilon^{3 / 2} \frac{\partial}{\partial \tau}-\epsilon^{1 / 2} v_{0} \frac{\partial}{\partial \xi}\right)\left(1+\epsilon n_{d 1}+\epsilon^{2} n_{d 2}+\epsilon^{3} n_{d 3}\right) \\
& +\left(\epsilon^{1 / 2} \frac{\partial}{\partial \xi}\right)\left[\left(1+\epsilon n_{d 1}+\epsilon^{2} n_{d 2}+\epsilon^{3} n_{d 3}\right)\right. \\
& \left.\cdot\left(u_{d 0}+\epsilon u_{d 1}+\epsilon^{2} u_{d 2}+\epsilon^{3} u_{d 3}\right)\right]+\left(\epsilon^{1 / 2} \frac{\partial}{\partial \eta}\right) \\
& \cdot\left[\left(1+\epsilon n_{d 1}+\epsilon^{2} n_{d 2}+\epsilon^{3} n_{d 3}\right)\right. \\
& \left.\cdot\left(\epsilon^{3 / 2} v_{d 1}+\epsilon^{5 / 2} v_{d 2}+\epsilon^{7 / 2} v_{d 3}\right)\right]+\left(\epsilon^{1 / 2} \frac{\partial}{\partial \zeta}\right) \\
& \cdot\left[\left(1+\epsilon n_{d 1}+\epsilon^{2} n_{d 2}+\epsilon^{3} n_{d 3}\right)\right. \\
& \left.\cdot\left(\epsilon^{3 / 2} w_{d 1}+\epsilon^{5 / 2} w_{d 2}+\epsilon^{7 / 2} w_{d 3}\right)\right]=0, \\
& \left(\epsilon^{3 / 2} \frac{\partial}{\partial \tau}-\epsilon^{1 / 2} v_{0} \frac{\partial}{\partial \xi}\right)\left(u_{d 0}+\epsilon u_{d 1}+\epsilon^{2} u_{d 2}+\epsilon^{3} u_{d 3}\right) \\
& +\left(u_{d 0}+\epsilon u_{d 1}+\epsilon^{2} u_{d 2}+\epsilon^{3} u_{d 3}\right) \cdot\left(\epsilon^{1 / 2} \frac{\partial}{\partial \xi}\right)\left(u_{d 0}\right. \\
& \left.+\epsilon u_{d 1}+\epsilon^{2} u_{d 2}+\epsilon^{3} u_{d 3}\right)+\left(\epsilon^{3 / 2} v_{d 1}+\epsilon^{5 / 2} v_{d 2}\right. \\
& \left.+\epsilon^{7 / 2} v_{d 3}\right)\left(\epsilon^{1 / 2} \frac{\partial}{\partial \eta}\right)\left(u_{d 0}+\epsilon u_{d 1}+\epsilon^{2} u_{d 2}+\epsilon^{3} u_{d 3}\right) \\
& +\left(\epsilon^{3 / 2} w_{d 1}+\epsilon^{5 / 2} w_{d 2}+\epsilon^{7 / 2} w_{d 3}\right)\left(\epsilon^{1 / 2} \frac{\partial}{\partial \zeta}\right)\left(u_{d 0}\right. \\
& \left.+\epsilon u_{d 1}+\epsilon^{2} u_{d 2}+\epsilon^{3} u_{d 3}\right)+\frac{T_{d}}{Z_{d 0}} \\
& \cdot \frac{1}{1+\epsilon n_{d 1}+\epsilon^{2} n_{d 2}+\epsilon^{3} n_{d 3}}\left(\epsilon^{1 / 2} \frac{\partial}{\partial \xi}\right)\left(1+\epsilon n_{d 1}\right. \\
& \left.+\epsilon^{2} n_{d 2}+\epsilon^{3} n_{d 3}\right)-\left(\epsilon^{1 / 2} \frac{\partial}{\partial \xi}\right)\left(\epsilon \phi_{1}+\epsilon^{2} \phi_{2}+\epsilon^{3} \phi_{3}\right) \\
& =-\left(\beta_{0}+\epsilon \beta_{1}\right)\left(u_{d 0}+\epsilon u_{d 1}+\epsilon^{2} u_{d 2}+\epsilon^{3} u_{d 3}\right)+g, \\
& \left(\epsilon^{3 / 2} \frac{\partial}{\partial \tau}-\epsilon^{1 / 2} v_{0} \frac{\partial}{\partial \xi}\right)\left(\epsilon^{3 / 2} v_{d 1}+\epsilon^{5 / 2} v_{d 2}+\epsilon^{7 / 2} v_{d 3}\right)
\end{aligned}
$$




$$
\begin{aligned}
& +\left(u_{d 0}+\epsilon u_{d 1}+\epsilon^{2} u_{d 2}+\epsilon^{3} u_{d 3}\right)\left(\epsilon^{1 / 2} \frac{\partial}{\partial \xi}\right) \cdot\left(\epsilon^{3 / 2} v_{d 1}\right. \\
& \left.+\epsilon^{5 / 2} v_{d 2}+\epsilon^{7 / 2} v_{d 3}\right)+\left(\epsilon^{3 / 2} v_{d 1}+\epsilon^{5 / 2} v_{d 2}+\epsilon^{7 / 2} v_{d 3}\right) \\
& \cdot\left(\epsilon^{1 / 2} \frac{\partial}{\partial \eta}\right)\left(\epsilon^{3 / 2} v_{d 1}+\epsilon^{5 / 2} v_{d 2}+\epsilon^{7 / 2} v_{d 3}\right)+\left(\epsilon^{3 / 2} w_{d 1}\right. \\
& \left.+\epsilon^{5 / 2} w_{d 2}+\epsilon^{7 / 2} w_{d 3}\right)\left(\epsilon^{1 / 2} \frac{\partial}{\partial \zeta}\right)\left(\epsilon^{3 / 2} v_{d 1}+\epsilon^{5 / 2} v_{d 2}\right. \\
& \left.+\epsilon^{7 / 2} v_{d 3}\right)+\frac{T_{d}}{Z_{d 0}} \\
& \cdot \frac{1}{1+\epsilon n_{d 1}+\epsilon^{2} n_{d 2}+\epsilon^{3} n_{d 3}}\left(\epsilon^{1 / 2} \frac{\partial}{\partial \eta}\right)\left(1+\epsilon n_{d 1}\right. \\
& \left.+\epsilon^{2} n_{d 2}+\epsilon^{3} n_{d 3}\right)-\left(\epsilon^{1 / 2} \frac{\partial}{\partial \eta}\right)\left(\epsilon \phi_{1}+\epsilon^{2} \phi_{2}+\epsilon^{3} \phi_{3}\right) \\
& =-\left(\epsilon \beta_{1}\right)\left(\epsilon^{3 / 2} v_{d 1}+\epsilon^{5 / 2} v_{d 2}+\epsilon^{7 / 2} v_{d 3}\right), \\
& \left(\epsilon^{3 / 2} \frac{\partial}{\partial \tau}-\epsilon^{1 / 2} v_{0} \frac{\partial}{\partial \xi}\right)\left(\epsilon^{3 / 2} w_{d 1}+\epsilon^{5 / 2} w_{d 2}+\epsilon^{7 / 2} w_{d 3}\right) \\
& +\left(u_{d 0}+\epsilon u_{d 1}+\epsilon^{2} u_{d 2}+\epsilon^{3} u_{d 3}\right)\left(\epsilon^{1 / 2} \frac{\partial}{\partial \xi}\right) \cdot\left(\epsilon^{3 / 2} w_{d 1}\right. \\
& \left.+\epsilon^{5 / 2} w_{d 2}+\epsilon^{7 / 2} w_{d 3}\right)+\left(\epsilon^{3 / 2} v_{d 1}+\epsilon^{5 / 2} v_{d 2}\right. \\
& \left.+\epsilon^{7 / 2} v_{d 3}\right)\left(\epsilon^{1 / 2} \frac{\partial}{\partial \eta}\right)\left(\epsilon^{3 / 2} w_{d 1}+\epsilon^{5 / 2} w_{d 2}+\epsilon^{7 / 2} w_{d 3}\right) \\
& +\left(\epsilon^{3 / 2} w_{d 1}+\epsilon^{5 / 2} w_{d 2}+\epsilon^{7 / 2} w_{d 3}\right)\left(\epsilon^{1 / 2} \frac{\partial}{\partial \zeta}\right)\left(\epsilon^{3 / 2} w_{d 1}\right. \\
& \left.+\epsilon^{5 / 2} w_{d 2}+\epsilon^{7 / 2} w_{d 3}\right)+\frac{T_{d}}{Z_{d 0}} \\
& \cdot \frac{1}{1+\epsilon n_{d 1}+\epsilon^{2} n_{d 2}+\epsilon^{3} n_{d 3}}\left(\epsilon^{1 / 2} \frac{\partial}{\partial \zeta}\right)\left(1+\epsilon n_{d 1}\right. \\
& \left.+\epsilon^{2} n_{d 2}+\epsilon^{3} n_{d 3}\right)-\left(\epsilon^{1 / 2} \frac{\partial}{\partial \zeta}\right)\left(\epsilon \phi_{1}+\epsilon^{2} \phi_{2}+\epsilon^{3} \phi_{3}\right) \\
& =-\left(\epsilon \beta_{1}\right)\left(\epsilon^{3 / 2} w_{d 1}+\epsilon^{5 / 2} w_{d 2}+\epsilon^{7 / 2} w_{d 3}\right), \\
& \left(\epsilon \frac{\partial^{2}}{\partial \xi^{2}}\right)\left(\epsilon \phi_{1}+\epsilon^{2} \phi_{2}+\epsilon^{3} \phi_{3}\right)+\left(\epsilon \frac{\partial^{2}}{\partial \eta^{2}}\right)\left(\epsilon \phi_{1}+\epsilon^{2} \phi_{2}\right. \\
& \left.+\epsilon^{3} \phi_{3}\right)+\left(\epsilon \frac{\partial^{2}}{\partial \zeta^{2}}\right)\left(\epsilon \phi_{1}+\epsilon^{2} \phi_{2}+\epsilon^{3} \phi_{3}\right)=\left(1+\epsilon n_{d 1}\right. \\
& \left.+\epsilon^{2} n_{d 2}+\epsilon^{3} n_{d 3}\right)+\left(\epsilon \phi_{1}+\epsilon^{2} \phi_{2}+\epsilon^{3} \phi_{3}\right) \text {. }
\end{aligned}
$$

In (6), letting

$$
T=\frac{T_{d}}{Z_{d 0}} \frac{1}{1+\epsilon n_{d 1}+\epsilon^{2} n_{d 2}+\epsilon^{3} n_{d 3}}\left(\epsilon^{1 / 2} \frac{\partial}{\partial \xi}\right)
$$

$$
\begin{aligned}
& \cdot\left(1+\epsilon n_{d 1}+\epsilon^{2} n_{d 2}+\epsilon^{3} n_{d 3}\right)=\frac{T_{d}}{Z_{d 0}}\left(\epsilon^{1 / 2} \frac{\partial}{\partial \xi}\right) \\
& \cdot\left[\ln \left(1+\epsilon n_{d 1}+\epsilon^{2} n_{d 2}+\epsilon^{3} n_{d 3}\right)\right],
\end{aligned}
$$

and using the Taylor's series, $T$ can be approximately rewritten as

$$
T=\frac{T_{d}}{Z_{d 0}}\left(\epsilon^{1 / 2} \frac{\partial}{\partial \xi}\right)\left(\epsilon n_{d 1}+\epsilon^{2} n_{d 2}+\epsilon^{3} n_{d 3}+\cdots\right) .
$$

A series of approximate equations for $\epsilon$ in the following form can be obtained

$$
\begin{aligned}
& \epsilon^{0}:-\beta_{0} u_{d 0}=g ; \\
& \epsilon^{1}: \beta_{1} u_{d 0}+\beta_{0} u_{d 1}=0 ; \\
& \epsilon^{3 / 2}:\left\{\begin{array}{l}
\left(u_{d 0}-v_{0}\right) \frac{\partial n_{d 1}}{\partial \xi}+\frac{\partial u_{d 1}}{\partial \xi}=0, \\
\left(u_{d 0}-v_{0}\right) \frac{\partial u_{d 1}}{\partial \xi}+\frac{T_{d}}{Z_{d}} \frac{\partial n_{d 1}}{\partial \xi}-\frac{\partial \phi_{1}}{\partial \xi}=0 ;
\end{array}\right. \\
& \epsilon^{2}:\left\{\begin{array}{l}
\beta_{0} u_{d 2}+\beta_{1} u_{d 1}=0 \\
\frac{\partial^{2} \phi_{1}}{\partial \xi^{2}}+\frac{\partial^{2} \phi_{1}}{\partial \eta^{2}}+\frac{\partial^{2} \phi_{1}}{\partial \zeta^{2}}=n_{d 2}+\phi_{2} ;
\end{array}\right. \\
& \epsilon^{5 / 2}:\left\{\begin{array}{l}
\left(u_{d 0}-v_{0}\right) \frac{\partial n_{d 2}}{\partial \xi}+\frac{\partial u_{d 2}}{\partial \xi}=-\frac{\partial n_{d 1}}{\partial \tau}-n_{d 1} \frac{\partial u_{d 1}}{\partial \xi}-u_{d 1} \frac{\partial n_{d 1}}{\partial \xi}, \\
\left(u_{d 0}-v_{0}\right) \frac{\partial u_{d 2}}{\partial \xi}-\frac{\partial \phi_{2}}{\partial \xi}+\frac{T_{d}}{Z_{d}} \frac{\partial n_{d 2}}{\partial \xi}=-\frac{\partial u_{d 1}}{\partial \tau}-u_{d 1} \frac{\partial u_{d 1}}{\partial \xi} .
\end{array}\right.
\end{aligned}
$$

Based on (9) and (10), we get the following relations

$$
\begin{aligned}
& \beta_{0}=\frac{g}{u_{d 0}}, \\
& \beta_{1}=\frac{-\beta_{0}\left(u_{d 0}-v_{0}\right)}{u_{d 0}} \phi_{1}=\frac{-\beta_{0}^{2}\left(u_{d 0}-v_{0}\right) \phi_{1}}{g} \phi_{1} .
\end{aligned}
$$

According to (11), the following relationship can be given as

$$
\left[\left(u_{d 0}-v_{0}\right)^{2}-\frac{T_{d}}{Z_{d}}\right] \frac{\partial n_{d 1}}{\partial \xi}+\frac{\partial \phi_{d 1}}{\partial \xi}=0 .
$$

Assuming that

$$
n_{d 1}=-\phi_{1} \text {, }
$$

we get

$$
u_{d 1}=\left(u_{d 0}-v_{0}\right) \phi_{1}
$$

$$
\frac{Z_{d 0}}{Z_{d 0}\left(u_{d 0}-v_{0}\right)^{2}-T_{d}}=1 \text {. }
$$

Combining (12), (13), (16), and (17) and letting $\phi_{1}=\phi$, we can obtain the following model:

$$
\frac{\partial \phi}{\partial \tau}+a_{1} \phi \frac{\partial \phi}{\partial \xi}+a_{2} \frac{\partial^{3} \phi}{\partial \xi^{3}}+a_{3} \frac{\partial^{3} \phi}{\partial \xi \partial \eta^{2}}+a_{4} \frac{\partial^{3} \phi}{\partial \xi \partial \zeta^{2}}=0,
$$


where

$$
\begin{aligned}
& a_{1}=\frac{3\left(g-\beta_{0} v_{0}\right)}{2 \beta_{0}}=\frac{3}{2} \sqrt{\frac{Z_{d 0}+T_{d}}{Z_{d 0}}}, \\
& a_{2}=a_{3}=a_{4}=-\frac{\beta_{0}}{2\left(g-\beta_{0} v_{0}\right)}=-\frac{1}{2} \sqrt{\frac{Z_{d 0}}{Z_{d 0}+T_{d}}} .
\end{aligned}
$$

When $a_{3}=a_{4}=0,(18)$ is the KdV equation. When $a_{4}=0$, (18) is the ZK equation. On the basis of $a_{3}\left(\partial^{3} \phi / \partial \xi \partial \eta^{2}\right)$ and $a_{4}\left(\partial^{3} \phi / \partial \xi \partial \zeta^{2}\right),(18)$ is a $(3+1)$-dimensional $\mathrm{ZK}$ equation. It is well known that the $\mathrm{KdV}$ equation reflects the propagation of dust acoustic waves along a line, and the $\mathrm{ZK}$ equation can describe the propagation of dust acoustic waves on one surface. Dust acoustic waves in dust plasma move around in space. Therefore, the $(3+1)$-dimensional $\mathrm{ZK}$ equation is more suitable to describe the dust acoustic waves in the real dust plasma.

\section{Derivation of the $(3+1)$-Dimensional Time-Space Fractional ZK (TSF-ZK) Equation}

Based on the above section, we get a $(3+1)$-dimensional ZK equation. In this section, the (3+1)-dimensional TSF-ZK equation is obtained by using the semi-inverse method and the fractional variational principle.

Firstly, let us introduce some basic fractional definitions.

Definition 1 (see [50]). The Riemann-Liouville fractional derivative operator is defined as

$$
D_{t}^{\alpha} u= \begin{cases}\frac{\partial_{m} u}{\partial t^{m}}, & \alpha=m \in N, \\ \frac{1}{\Gamma(m-\alpha)} \frac{\partial_{m}}{\partial t^{m}} \int_{0}^{t} \frac{u(\tau, x)}{(t-\tau)^{\alpha+1-m}} d \tau, & m-1<\alpha<m, m \in N .\end{cases}
$$

Definition 2 (see [50]). The modified Riemann-Liouville fractional derivative operator is defined as

$$
\begin{aligned}
D_{t}^{\alpha} f(t)=\frac{1}{\Gamma(1-\alpha)} \frac{d}{d t}\left[\int_{a}^{t} \frac{f(\tau)-f(a)}{(t-\tau)^{\alpha}} d \tau\right] & \\
& 0 \leq \alpha<1 .
\end{aligned}
$$

Letting $\phi=Q_{\xi}$, where $Q(\tau, \xi, \eta, \zeta)$ is a potential function, then the (18) can be written as

$$
Q_{\xi \tau}+a_{1} Q_{\xi} Q_{\xi \xi}+a_{2} Q_{\xi \xi \xi \xi}+a_{3} Q_{\xi \xi \eta \eta}+a_{4} Q_{\xi \xi \zeta \zeta}=0 .
$$

The functional of the above potential equation can be given as

$$
J(Q)=\int_{R} d \xi \int_{R} d \eta \int_{R} d \zeta \int_{T} d \tau\left[Q(\tau, \xi, \eta, \zeta)\left(b_{1} Q_{\xi \tau}+b_{2} a_{1} Q_{\xi} Q_{\xi \xi}+b_{3} a_{2} Q_{\xi \xi \xi \xi}+b_{4} a_{3} Q_{\xi \xi \eta \eta}+b_{5} a_{4} Q_{\xi \xi \zeta \zeta}\right)\right]
$$

where $d_{i}(i=1,2,3,4,5)$ are Lagrangian multipliers, which can be obtained later, and $\xi \in R$. Integrating (23) by parts and letting $\left.Q_{\tau}\right|_{T}=\left.Q_{\xi}\right|_{R}=\left.Q_{\eta}\right|_{R}=\left.Q_{\zeta}\right|_{R}=\left.Q_{\xi}\right|_{T}=\left.Q_{\xi \xi \xi}\right|_{R}=$ $\left.Q_{\xi \eta \eta}\right|_{R}=\left.Q_{\xi \zeta \zeta}\right|_{R}=0$, we can get

$$
\begin{gathered}
J(Q)=\iiint_{V} d \xi d \eta d \zeta \int_{R}\left(-b_{1} Q_{\tau} Q_{\xi}-\frac{1}{2} b_{2} a_{1} q_{\xi}^{3}\right. \\
\left.+b_{3} a_{2} Q_{\xi \xi}^{2}+b_{4} a_{3} Q_{\xi \eta}^{2}++b_{5} a_{4} Q_{\xi \zeta}^{2}\right) d \tau,
\end{gathered}
$$

where $V=[R \times R \times R]$. Using the variation of (24) and integrating each term by parts, we get

$$
\begin{aligned}
& F\left(\tau, \xi, \eta, \zeta, Q, Q_{\tau}, Q_{\xi}, Q_{\xi \xi}, Q_{\xi \eta}, Q_{\xi \zeta}\right) \\
& =-\frac{\partial}{\partial \tau}\left(\frac{\partial F}{\partial Q_{\tau}}\right)-\frac{\partial}{\partial \xi}\left(\frac{\partial F}{\partial Q_{\xi}}\right)+\frac{\partial^{2}}{\partial \xi^{2}}\left(\frac{\partial F}{\partial Q_{\xi \xi}}\right)
\end{aligned}
$$

$$
\begin{aligned}
& +\frac{\partial^{2}}{\partial \xi \eta}\left(\frac{\partial F}{\partial Q_{\xi \eta}}\right)+\frac{\partial^{2}}{\partial \xi \zeta}\left(\frac{\partial F}{\partial Q_{\xi \zeta}}\right) \\
= & b_{1} Q_{\xi \tau}+3 b_{2} a_{1} Q_{\xi} Q_{\xi \xi}+2 b_{3} a_{2} Q_{\xi \xi \xi \xi}+2 b_{4} a_{3} Q_{\xi \xi \eta \eta} \\
& +2 b_{5} a_{4} Q_{\xi \xi \zeta \zeta} .
\end{aligned}
$$

Thus, the following comparison expression is given as

$$
\begin{aligned}
b_{1} Q_{\xi \tau} & +3 b_{2} a_{1} Q_{\xi} Q_{\xi \xi}+2 b_{3} a_{2} Q_{\xi \xi \xi \xi}+2 b_{4} a_{3} Q_{\xi \xi \eta \eta} \\
& +2 b_{5} a_{4} Q_{\xi \xi \zeta \zeta}=0 .
\end{aligned}
$$

At this point, (26) is equivalent to (22), so the Lagrangian multipliers are as follows:

$$
b_{1}=\frac{1}{2}
$$




$$
\begin{aligned}
& b_{2}=\frac{1}{3}, \\
& b_{3}=b_{4}=b_{5}=\frac{1}{2} .
\end{aligned}
$$

The Lagrangian form of the (3+1)-dimensional ZK equation can be written as follows:

$$
\begin{aligned}
& L\left(Q_{\tau}, Q_{\xi}, Q_{\xi \xi}, Q_{\xi \eta}, Q_{\xi \zeta}\right) \\
& \quad=-\frac{1}{2} Q_{\tau} Q_{\xi}-\frac{1}{6} a_{1} Q_{\xi}^{3}+\frac{1}{2} Q_{\xi \xi}^{2}+\frac{1}{2} Q_{\xi \eta}^{2}+\frac{1}{2} Q_{\xi \eta}^{2} .
\end{aligned}
$$

Similarly, the following Lagrangian form of the $(3+1)$ dimensional TSF-ZK equation can be obtained

$$
\begin{aligned}
F( & \left.D_{\tau}^{\alpha} Q, D_{\xi}^{\beta} Q, D_{\xi \xi}^{\beta \beta} Q, D_{\xi}^{\beta} D_{\eta}^{\gamma} Q, D_{\xi}^{\beta} D_{\zeta}^{\theta} Q\right) \\
= & -\frac{1}{2} D_{\tau}^{\alpha} Q D_{\xi}^{\beta} Q-\frac{1}{6} a_{1}\left(D_{\xi}^{\beta} Q\right)^{3}+\frac{1}{2}\left(D_{\xi \xi}^{\beta \beta} Q\right)^{3} \\
& +\frac{1}{2}\left(D_{\xi}^{\beta} D_{\eta}^{\gamma} Q\right)^{2}+\frac{1}{2} D_{\xi}^{\beta} D_{\zeta}^{\theta} Q,
\end{aligned}
$$

where $\alpha, \beta, \beta$, and $\theta$ are fractional order values. Thereby the functional of $(3+1)$-dimensional TSF-ZK equation is given as follows:

$$
\begin{aligned}
& J_{F}(Q)=\iiint_{V}(d \xi)^{\beta}(d \eta)^{\gamma}(d \eta)^{\eta} \\
& \quad \cdot \int_{T} F\left(D_{\tau}^{\alpha} Q, D_{\xi}^{\beta} Q, D_{\xi \xi}^{\beta \beta} Q, D_{\xi}^{\beta} D_{\eta}^{\gamma} Q, D_{\xi}^{\beta} D_{\zeta}^{\theta} Q\right) \\
& \cdot(d \tau)^{\alpha},
\end{aligned}
$$

where

$$
\int_{a}^{t} f(\tau)(d \tau)^{\alpha}=\alpha \int_{a}^{t} f(\tau)(t-\tau)^{\alpha} d \tau .
$$

Using the fractional integration by parts

$$
\begin{array}{r}
\int_{a}^{b} f(t) D_{t}^{\alpha} g(t)(d \tau)^{\alpha} \\
=\Gamma(1+\alpha)\left[\left.g(t) f(t)\right|_{a} ^{b}-\int_{a}^{b} g(t) D_{t}^{\alpha} f(t)(d \tau)^{\alpha}\right], \\
f(t), g(t) \in[a, b],
\end{array}
$$

optimizing the above functional (30), and letting $\delta J_{F}(Q)=0$, we can get the following Euler-Lagrange equation of $(3+1)$ dimensional TSF-ZK equation

$$
\begin{array}{r}
-D_{\tau}^{\alpha}\left(\frac{\partial F}{\partial D_{\tau}^{\alpha} Q}\right)-D_{\xi}^{\beta}\left(\frac{\partial F}{\partial D_{\xi}^{\beta} Q}\right)+D_{\xi}^{\beta \beta}\left(\frac{\partial F}{\partial D_{\xi}^{\beta \beta} Q}\right) \\
+D_{\xi}^{\beta} D_{\eta}^{\gamma}\left(\frac{\partial F}{\partial D_{\xi}^{\beta} D_{\eta}^{\gamma} Q}\right)+D_{\xi}^{\beta} D_{\zeta}^{\theta}\left(\frac{\partial F}{\partial D_{\xi}^{\beta} D_{\zeta}^{\theta} Q}\right)
\end{array}
$$

According to (29), (33) can be rewritten as

$$
\begin{array}{r}
D_{\tau}^{\alpha}\left(D_{\xi}^{\beta} Q\right)+a_{1} D_{\xi}^{\beta} Q D_{\xi}^{\beta \beta} Q+a_{2} D_{\xi}^{\beta \beta \beta \beta} Q \\
+a_{3} D_{\xi}^{\beta \beta} D_{\eta}^{\gamma \gamma} Q+a_{4} D_{\xi}^{\beta \beta} D_{\eta}^{\theta \theta} Q=0 .
\end{array}
$$

Letting $D_{\xi}^{\beta} Q=\phi(\xi, \eta, \zeta, \tau)$, we get

$$
\begin{aligned}
& D_{\tau}^{\alpha} \phi+a_{1} \phi D_{\xi}^{\beta} \phi+a_{2} D_{\xi}^{\beta \beta \beta} \phi+a_{3} D_{\xi}^{\beta} D_{\eta}^{\gamma \gamma} \phi+a_{4} D_{\xi}^{\beta} D_{\eta}^{\theta \theta} \phi \\
& \quad=0 .
\end{aligned}
$$

Equation (35) is a new model, namely, the $(3+1)$ dimensional TSF-ZK equation. When $\alpha=\beta=\gamma=\theta=$ 1 , (35) is the integer order $(3+1)$-dimensional $\mathrm{ZK}$ equation. This shows that the integer order model is the special type of fractional model. The fractional derivatives of the $(3+1)$ dimensional TSF-ZK equation are related to the dust acoustic waves propagation with fractal properties. Equation (35) can describe fractal processes of the dust acoustic waves. The fractional order model could simulate various real plasma environments more adequately than the integer order model and provide an excellent tool for the description of dynamical processes. Therefore, (3+1)-dimensional TSF-ZK equation can better describe the dust acoustic waves in the real dust plasma.

\section{Conservation Law of the (3+1)-Dimensional TSF-ZK Equation}

In the last section, we extend the integral order equations describing dust acoustic waves to the fractional order of timespace and obtain some new properties of dust acoustic waves propagation. In this section, in order to research the energy changes during the propagation of dust acoustic waves, we use the Lie symmetry method to obtain multiple conservation law of the $(3+1)$-dimensional TSF-ZK equation.

Considering that, under one-parameter Lie group of point transformations, (35) is invariant with the dependent and independent variables, the transformations are given by

$$
\begin{aligned}
& \bar{\xi} \longrightarrow \xi+\varepsilon l(\xi, \eta, \zeta, \tau)+O\left(\varepsilon^{2}\right), \\
& \bar{\eta} \longrightarrow \eta+\varepsilon \kappa(\xi, \eta, \zeta, \tau)+O\left(\varepsilon^{2}\right), \\
& \bar{\zeta} \longrightarrow \zeta+\varepsilon v(\xi, \eta, \zeta, \tau)+O\left(\varepsilon^{2}\right), \\
& \bar{\tau} \longrightarrow \tau+\varepsilon \sigma(\xi, \eta, \zeta, \tau)+O\left(\varepsilon^{2}\right), \\
& \bar{\phi} \longrightarrow \phi+\varepsilon \Phi(\xi, \eta, \zeta, \tau)+O\left(\varepsilon^{2}\right),
\end{aligned}
$$




$$
\begin{aligned}
D_{\tau}^{\alpha} \bar{\phi} & \longrightarrow D_{\tau}^{\alpha} \phi+\varepsilon \Phi_{\alpha}^{\tau}+O\left(\varepsilon^{2}\right), \\
D_{\xi}^{\beta} \bar{\phi} & \longrightarrow D_{\xi}^{\beta} \phi+\varepsilon \Phi_{\beta}^{\xi}+O\left(\varepsilon^{2}\right), \\
D_{\xi}^{\beta \beta \beta} \bar{\phi} & \longrightarrow D_{\xi}^{\beta \beta \beta} \phi+\varepsilon \Phi_{\beta}^{\xi \xi \xi}+O\left(\varepsilon^{2}\right), \\
D_{\tau}^{\alpha} D_{\xi}^{\beta \beta} \bar{\phi} & \longrightarrow D_{\tau}^{\alpha} D_{\xi}^{\beta \beta} \phi+\varepsilon \Phi_{\alpha, \beta}^{\tau \xi \xi}+O\left(\varepsilon^{2}\right), \\
D_{\tau}^{\alpha} D_{\zeta}^{\theta \theta} \bar{\phi} & \longrightarrow D_{\tau}^{\alpha} D_{\zeta}^{\theta \theta} \phi+\varepsilon \Phi_{\alpha, \zeta}^{\tau \theta \theta}+O\left(\varepsilon^{2}\right),
\end{aligned}
$$

where $\iota, \kappa, v, \sigma$ are infinitesimal function of the transformations, $\varepsilon<<1$ is the group parameter, and $\Phi_{\alpha}^{\tau}, \Phi_{\beta}^{\xi}, \Phi_{\beta}^{\xi \xi \xi}$, $\Phi_{\alpha, \beta}^{\tau \xi \xi}, \Phi_{\alpha, \zeta}^{\tau \theta \theta}$ are extended infinitesimal functions in the following explicit expression:

$$
\begin{aligned}
\Phi_{\alpha}^{\tau}= & D_{\tau}^{\alpha}(\Phi)+\iota D_{\tau}^{\alpha}\left(\phi_{\xi}\right)-D_{\tau}^{\alpha}\left(\iota \phi_{\xi}\right)+\kappa D_{\tau}^{\alpha}\left(\phi_{\eta}\right) \\
& -D_{\tau}^{\alpha}\left(\kappa \phi_{\eta}\right)+v D_{\tau}^{\alpha}\left(\phi_{\zeta}\right)-D_{\tau}^{\alpha}\left(v \phi_{\zeta}\right) \\
& +D_{\tau}^{\alpha}\left(D_{\tau}(\sigma) \phi\right)-D_{\tau}^{\alpha+1}(\sigma \phi) \\
& +\sigma D_{\tau}^{\alpha+1}(\phi), \\
\Phi_{\beta}^{\xi}= & D_{\xi}^{\beta}(\Phi)+D_{\xi}^{\beta}(\phi) D_{\xi}(\iota)-D_{\eta}^{\gamma}(\phi) D_{\xi}(\kappa) \\
& -D_{\zeta}^{\theta}(\phi) D_{\xi}(v), \\
\Phi_{\beta}^{\xi \xi \xi}= & D_{\xi}^{\beta}\left(\Phi_{\beta}^{\xi \xi}\right)-\phi_{\xi \xi \xi} D_{\xi}^{\beta}(\iota)-\phi_{\xi \xi \eta} D_{\xi}^{\beta}(\kappa) \\
& -\phi_{\xi \xi \zeta} D_{\xi}^{\beta}(v)-\phi_{\xi \xi \tau} D_{\xi}^{\beta}(\sigma), \\
\Phi_{\alpha, \beta}^{\tau \xi \xi}= & D_{\xi}^{\beta}\left(\Phi_{\beta}^{\eta \eta}\right)-\phi_{\xi \eta \xi} D_{\xi}^{\beta}(\iota)-\phi_{\xi \eta \eta} D_{\xi}^{\beta}(\kappa)
\end{aligned}
$$

$$
\begin{aligned}
& -\phi_{\xi \eta \zeta} D_{\xi}^{\beta}(v)-\phi_{\xi \eta \tau} D_{\xi}^{\beta}(\sigma), \\
\Phi_{\alpha, \zeta}^{\tau \theta \theta}= & D_{\xi}^{\beta}\left(\Phi_{\beta}^{\zeta \zeta}\right)-\phi_{\xi \zeta \zeta} D_{\xi}^{\beta}(\iota)-\phi_{\xi \zeta \eta} D_{\xi}^{\beta}(\kappa) \\
& -\phi_{\xi \zeta \zeta} D_{\xi}^{\beta}(v)-\phi_{\xi \zeta \tau} D_{\xi}^{\beta}(\sigma),
\end{aligned}
$$

where $D_{\tau}$ and $D_{\xi}$ are the total derivative operators as follows:

$$
\begin{aligned}
D_{\tau}= & \frac{\partial}{\partial \tau}+\phi_{\tau} \frac{\partial}{\partial \tau}+\phi_{\tau \tau} \frac{\partial}{\partial \phi_{\tau}}+\phi_{\xi \tau} \frac{\partial}{\partial \phi_{\xi}}+\phi_{\eta \tau} \frac{\partial}{\partial \phi_{\eta}} \\
& +\phi_{\zeta \tau} \frac{\partial}{\partial \phi_{\zeta}}+\cdots, \\
D_{\xi}= & \frac{\partial}{\partial \xi}+\phi_{\xi} \frac{\partial}{\partial \xi}+\phi_{\xi \xi} \frac{\partial}{\partial \phi_{\xi}}+\phi_{\tau \xi} \frac{\partial}{\partial \phi_{\tau}}+\phi_{\eta \xi} \frac{\partial}{\partial \phi_{\eta}} \\
& +\phi_{\zeta \xi} \frac{\partial}{\partial \phi_{\zeta}}+\cdots .
\end{aligned}
$$

Applying the generalized Leibnitz rule and the chain rule, the extended symmetry operator $\Phi$ can be introduced in the following form:

$$
\begin{aligned}
\Phi_{\alpha}^{\tau} & =\frac{\partial^{\alpha} \Phi}{\partial \tau^{\alpha}}+\left(\Phi_{\phi}-\alpha D_{\tau}^{\sigma}\right) \frac{\partial^{\alpha} \phi}{\partial \tau^{\alpha}}-\phi \frac{\partial^{\alpha} \Phi_{\phi}}{\partial \tau^{\alpha}} \\
& +\sum_{n=1}^{\infty}\left[\left(\begin{array}{c}
\alpha \\
n
\end{array}\right) \frac{\partial^{\alpha} \Phi_{\phi}}{\partial \tau^{\alpha}}-\left(\begin{array}{c}
\alpha \\
n+1
\end{array}\right) D_{\tau}^{n+1}(\sigma)\right] D_{\tau}^{\alpha-n} \\
& -\sum_{n=1}^{\infty}\left(\begin{array}{c}
\alpha \\
n
\end{array}\right)\left[D_{\tau}^{n}(\iota) D_{\tau}^{\alpha-n}\left(\phi_{\xi}\right)+D_{\tau}^{n}(\kappa) D_{\tau}^{\alpha-n}\left(\phi_{\eta}\right)\right. \\
& \left.+D_{\tau}^{n}(v) D_{\tau}^{\alpha-n}\left(\phi_{\zeta}\right)\right]+R_{\tau},
\end{aligned}
$$

where

$$
R_{\tau}=\sum_{n=2}^{\infty} \sum_{m=2}^{n} \sum_{k=2}^{m} \sum_{r=0}^{k-1}\left[\left(\begin{array}{l}
\alpha \\
n
\end{array}\right)\left(\begin{array}{l}
n \\
m
\end{array}\right)\left(\begin{array}{l}
k \\
r
\end{array}\right) \frac{1}{k !} \frac{\tau^{n-\alpha}}{\Gamma(n+1-\alpha)}(-\phi)^{r} \frac{\partial^{\phi}}{\partial \tau^{\phi}}(\phi)^{k-r} \frac{\partial^{n-m+k} \Phi}{\partial \tau^{n-m} \partial \phi^{k}}\right] .
$$

Similarly, we also have the following equation:

$$
\begin{aligned}
\Phi_{\beta}^{\xi} & =\frac{\partial^{\beta} \Phi}{\partial \xi^{\beta}}+\left(\Phi_{\phi}-\beta D_{\xi}^{\iota}\right) \frac{\partial^{\beta} \phi}{\partial \xi^{\beta}}-\phi \frac{\partial^{\beta} \Phi_{\phi}}{\partial \xi^{\beta}} \\
& +\sum_{n=1}^{\infty}\left[\left(\begin{array}{c}
\beta \\
n
\end{array}\right) \frac{\partial^{\beta} \Phi_{\phi}}{\partial \xi^{\beta}}-\left(\begin{array}{c}
\beta \\
n+1
\end{array}\right) D_{\xi}^{n+1}(\iota)\right] D_{\xi}^{\beta-n}
\end{aligned}
$$

$$
\begin{aligned}
& -\sum_{n=1}^{\infty}\left(\begin{array}{l}
\beta \\
n
\end{array}\right)\left[D_{\xi}^{n}(\sigma) D_{\xi}^{\beta-n}\left(\phi_{\tau}\right)+D_{\xi}^{n}(\kappa) D_{\xi}^{\beta-n}\left(\phi_{\eta}\right)\right. \\
& \left.+D_{\xi}^{n}(v) D_{\xi}^{\beta-n}\left(\phi_{\zeta}\right)\right]+R_{\xi},
\end{aligned}
$$

where

$$
R_{\xi}=\sum_{n=2}^{\infty} \sum_{m=2}^{n} \sum_{k=2}^{m} \sum_{r=0}^{k-1}\left[\left(\begin{array}{l}
\beta \\
n
\end{array}\right)\left(\begin{array}{l}
n \\
m
\end{array}\right)\left(\begin{array}{l}
k \\
r
\end{array}\right) \frac{1}{k !} \frac{\xi^{n-\beta}}{\Gamma(n+1-\beta)}(-\phi)^{r} \frac{\partial^{\phi}}{\partial \xi^{\phi}}(\phi)^{k-r} \frac{\partial^{n-m+k} \Phi}{\partial \xi^{n-m} \partial \phi^{k}}\right]
$$


Introducing the Lie algebra $N$ associated with (35) is composed of the following infinitesimal generator

$$
N=\iota \frac{\partial}{\partial \xi}+\kappa \frac{\partial}{\partial \eta}+v \frac{\partial}{\partial \zeta}+\Phi \frac{\partial}{\partial \phi} .
$$

Applying the infinitesimal transformations, the (35) is invariable that results in the invariance conditions given as

$$
\left.\operatorname{Pr}^{(\alpha, \beta, \gamma, \theta, 4)} N(\Delta)\right|_{\Delta=0}=0,
$$

where $\Delta$ represents the (3+1)-dimensional TSF-ZK equation.

We get following symmetry determining equation by using the third prolongation to (35)

$$
\begin{aligned}
\Phi_{\alpha}^{\tau} & +a_{1} \Phi D_{\xi}^{\beta} \phi+a_{1} \Phi_{\beta}^{\xi}+a_{2} \Phi_{\beta}^{\xi \xi \xi}+a_{3} \Phi_{\beta, \gamma}^{\xi \eta \eta}+a_{4} \Phi_{\beta, \theta}^{\xi \zeta \zeta} \\
& =0 .
\end{aligned}
$$

Substituting (37), (38), (39), and (41) into this equation, letting the same coefficients of derivatives to zero, and then solving the series of determining equations, we can obtain the following infinitesimal functions

$$
\begin{aligned}
\sigma & =\frac{b_{1} \tau}{\alpha}+b_{2}, \\
\iota & =\frac{b_{1} \xi}{\beta}+b_{3}, \\
\kappa & =\frac{b_{1} \eta}{2 \gamma}+b_{4}, \\
v & =\frac{b_{1} \zeta}{2 \theta}+b_{5}, \\
\Phi & =b_{1}\left(\frac{3-\beta-\alpha}{\beta+\alpha}\right) \phi,
\end{aligned}
$$

where $b_{i}, i=1 \cdots 5$, are arbitrary constants, since the corresponding infinitesimal generators can be expressed as

$$
\begin{aligned}
N_{1}= & \frac{\partial}{\partial \xi}, \\
N_{2}= & \frac{\partial}{\partial \eta}, \\
N_{3}= & \frac{\partial}{\partial \zeta}, \\
N_{4}= & \frac{\partial}{\partial \tau}, \\
N_{5}= & \frac{\tau}{\alpha} \frac{\partial}{\partial \tau}+\frac{\xi}{\beta} \frac{\partial}{\partial \xi}+\frac{\eta}{2 \gamma} \frac{\partial}{\partial \eta}+\frac{\zeta}{2 \theta} \frac{\partial}{\partial \zeta} \\
& +\left(\frac{3-\beta-\alpha}{\beta+\alpha}\right) \phi \frac{\partial}{\partial \phi} .
\end{aligned}
$$

The conserved vector of (35) is $C=\left(C^{\tau}, C^{\xi}, C^{\eta}, C^{\zeta}\right)$ which satisfies the conservation equation

$$
D_{\tau}\left(C^{\tau}\right)+D_{\xi}\left(C^{\xi}\right)+D_{\eta}\left(C^{\eta}\right)+D_{\zeta}\left(C^{\zeta}\right)=0 .
$$

A formal Lagrangian for the (3+1)-dimensional TSF-ZK equation is given as

$$
\begin{aligned}
L_{a} & =\rho(\xi, \eta, \zeta, \tau)\left(D_{\tau}^{\alpha} \phi+a_{1} \phi D_{\xi}^{\beta} \phi+a_{2} D_{\xi}^{\beta \beta \beta} \phi\right. \\
& \left.+a_{3} D_{\xi}^{\beta} D_{\eta}^{\gamma \gamma} \phi+a_{4} D_{\xi}^{\beta} D_{\eta}^{\theta \theta} \phi\right)
\end{aligned}
$$

where $\rho(\xi, \eta, \zeta, \tau)$ is the new dependent variable.

In the considered linear case, the adjoint equation to (35) as the Euler-Lagrange equation is represented as

$$
F^{*}=\frac{\delta L_{a}}{\delta \phi}=0 .
$$

Here $\delta / \delta \phi$ is the Euler-Lagrange operator which is defined as

$$
\begin{aligned}
\frac{\delta}{\delta \phi}= & \frac{\partial}{\partial \phi}+\left(D_{\tau}^{\alpha}\right)^{*} \frac{\partial}{\partial D_{\tau}^{\alpha}}+\left(D_{\xi}^{\beta}\right)^{*} \frac{\partial}{\partial D_{\xi}^{\beta} \phi} \\
& -\left(D_{\xi}^{\beta \beta \beta}\right)^{*} \frac{\partial}{\partial D_{\xi}^{\beta \beta \beta} \phi}-\left(D_{\xi}^{\beta} D_{\eta}^{\gamma \gamma}\right)^{*} \frac{\partial}{\partial D_{\xi}^{\beta} D_{\eta}^{\gamma \gamma} \phi} \\
& -\left(D_{\xi}^{\beta} D_{\zeta}^{\theta \theta}\right)^{*} \frac{\partial}{\partial D_{\xi}^{\beta} D_{\zeta}^{\theta \theta} \phi},
\end{aligned}
$$

where $\left(D_{\tau}^{\alpha}\right)^{*},\left(D_{\xi}^{\beta \beta \beta}\right)^{*},\left(D_{\xi}^{\beta} D_{\eta}^{\gamma \gamma}\right)^{*}$, and $\left(D_{\xi}^{\beta} D_{\zeta}^{\theta \theta}\right)^{*}$ are the adjoint operators.

Hence, the adjoint equation (50) can be rewritten as

$$
\begin{aligned}
F^{*}= & \left(D_{\tau}^{\alpha}\right)^{*} \rho+a_{1} \phi\left(D_{\xi}^{\beta}\right)^{*} \rho-a_{2}\left(D_{\xi}^{\beta \beta \beta}\right)^{*} \rho \\
& -a_{3}\left(D_{\xi}^{\beta} D_{\eta}^{\gamma \gamma}\right)^{*} \rho-a_{4}\left(D_{\xi}^{\beta} D_{\eta}^{\theta \theta}\right)^{*} \rho=0 .
\end{aligned}
$$

We introduce the Lie characteristic function $W$ for generator $N$ as follows:

$$
W=\Phi-\iota \phi_{\xi}-\kappa \phi_{\eta}-v \phi_{\zeta}-\sigma \phi_{\tau} .
$$

The fractional Noether operator for the variables $\tau, \xi, \eta$, and $\zeta$ are given by

$$
\begin{aligned}
C^{\tau} & =\sum_{k=0}^{n-1}(-1)^{k} D_{\tau}^{\alpha-1-k}(W) D_{\tau}^{k}\left(\frac{\partial L_{a}}{\partial\left(D_{\tau}^{\alpha} \phi\right)}\right)-(-1)^{n} \\
& \cdot J\left(W, D_{\tau}^{n}\left(\frac{\partial L_{a}}{\partial\left(D_{\tau}^{\alpha} \phi\right)}\right)\right), \\
C^{\xi} & =\sum_{k=0}^{m-1}(-1)^{k} D_{\xi}^{\beta-1-k}(W) D_{\xi}^{k}\left(\frac{\partial L_{a}}{\partial D_{\xi}^{\beta} \phi}+D_{\xi}^{\beta \beta} \frac{\partial L_{a}}{\partial D_{\xi}^{\beta \beta \beta} \phi}\right. \\
& \left.+D_{\eta}^{\gamma \gamma} \frac{\partial L_{a}}{\partial D_{\xi}^{\beta} D_{\eta}^{\gamma \gamma} \phi}+D_{\zeta}^{\theta \theta} \frac{\partial L_{a}}{\partial D_{\xi}^{\beta} D_{\zeta}^{\theta \theta} \phi}\right)-(-1)^{m}
\end{aligned}
$$




$$
\begin{aligned}
\cdot & J\left(W, D_{\xi}^{m}\left(\frac{\partial L_{a}}{\partial D_{\xi}^{\beta} \phi}+D_{\xi}^{\beta \beta} \frac{\partial L_{a}}{\partial D_{\xi}^{\beta \beta \beta} \phi}\right.\right. \\
+ & \left.\left.D_{\eta}^{\gamma \gamma} \frac{\partial L_{a}}{\partial D_{\xi}^{\beta} D_{\eta}^{\gamma \gamma} \phi}+D_{\zeta}^{\theta \theta} \frac{\partial L_{a}}{\partial D_{\xi}^{\beta} D_{\zeta}^{\theta \theta} \phi}\right)\right), \\
C^{\eta}= & \sum_{k=0}^{l-1}(-1)^{k} D_{\eta}^{\gamma-1-k}(W) D_{\eta}^{k}\left(D_{\xi}^{\beta} \frac{\partial L_{a}}{\partial D_{\eta}^{\gamma \gamma} \phi}\right)-(-1)^{l} \\
\cdot & J\left(W, D_{\eta}^{l}\left(D_{\xi}^{\beta} \frac{\partial L_{a}}{\partial D_{\eta}^{\gamma \gamma} \phi}\right)\right), \\
C^{\zeta}= & \sum_{k=0}^{s-1}(-1)^{k} D_{\zeta}^{\theta-1-k}(W) D_{\zeta}^{k}\left(D_{\xi}^{\beta} \frac{\partial L_{a}}{\partial D_{\zeta}^{\theta \theta} \phi}\right)-(-1)^{s} \\
& \cdot J\left(W, D_{\zeta}^{l}\left(D_{\xi}^{\beta} \frac{\partial L_{a}}{\partial D_{\zeta}^{\theta \theta} \phi}\right)\right),
\end{aligned}
$$

where

$$
\begin{aligned}
n & =[\alpha]+1, \\
m & =[\beta]+1, \\
l & =[\gamma]+1, \\
s & =[\theta]+1,
\end{aligned}
$$

and $J$ is an integral equation.

Consequently, the conservation law of the $(3+1)$ dimensional TSF-ZK equation is obtained. This indicates that the dust acoustic wave described by this new model is conserved in energy during its propagation, no matter in the fractal process or in the interaction.

\section{The Solution of the $(3+1)$-Dimensional TSF-ZK Equation}

In this section, we seek the exact solution of $(3+1)$ dimensional TSF-ZK equation by using the extended Kudryashov method. Firstly, for a given nonlinear partial differential equation

$$
\begin{aligned}
& F\left(\phi, \phi_{\tau}, \phi_{\xi}, \phi_{\eta}, \phi_{\zeta}, \ldots, D_{\tau}^{\alpha} \phi, D_{\xi}^{\beta} \phi, D_{\eta}^{\gamma} \phi, D_{\zeta}^{\theta} \phi, \ldots\right) \\
& \quad=0,
\end{aligned}
$$

we present the main solving process by using the extended Kudryashov method [47].

Step 1. We introduce the traveling wave solutions of the given nonlinear partial differential equation by making the following transformations:

$$
\begin{aligned}
& \phi(\xi, \eta, \zeta, \ldots, \tau)=\phi(E), \\
& E=\frac{k_{1} \tau^{\alpha}}{\Gamma(1+\alpha)}+\frac{k_{2} \xi^{\beta}}{\Gamma(1+\beta)}+\frac{k_{3} \eta^{\gamma}}{\Gamma(1+\gamma)}+\frac{k_{4} \zeta^{\theta}}{\Gamma(1+\theta)}+\cdots,
\end{aligned}
$$

where $k_{i}(i=1,2,3, \cdots)$ are arbitrary constants. Thus, the nonlinear partial differential equation reduces to a nonlinear ordinary differential equation as follows:

$$
G\left(\phi, \phi_{E}, \phi_{E E}, \phi_{E E E}, \cdots\right)=0 .
$$

Step 2. We let the new nonlinear ordinary differential equation have the following solution:

$$
\phi(E)=\sum_{i=0}^{N} c_{i} P^{i}(E), \quad c_{N} \neq 0,
$$

where $\phi(E)= \pm 1 / \sqrt{1 \pm e^{2 E}}$ and the function $P$ satisfies the following equation:

$$
P_{E}(E)=P^{3}(E)-P(E) .
$$

Step 3. We assume that the solution of (58) can be given in the following form:

$$
\phi=c_{N} P^{N}+c_{N-1} P^{N-1}+\cdots .
$$

In order to determine the value of the pole order $N$, we balance the highest order nonlinear terms $\phi^{l}(E) \phi^{(s)}(E)$ and the highest order linear terms $\left(\phi^{(p)}(E)\right)^{r}$ analogously. In the traditional method [53], we assume that the value of the pole order of $\phi(E)$ is $o(\phi(E))=N$, so the values of the pole order of the other terms are as follows:

$$
\begin{gathered}
o\left(\phi^{l}(E) \phi^{(s)}(E)\right)=(l+1) N+s, \\
o\left(\left(\phi^{(p)}(E)\right)^{r}\right)=(N+p) r .
\end{gathered}
$$

Letting $o\left(\phi^{l}(E) \phi^{(s)}(E)\right)=o\left(\phi^{(p)}(E)\right)$, we get $N$. However, there are some differences in the extended Kudryashov method. The value of the pole order $N$ is defined as

$$
N=\frac{2(s-r p)}{r-l-1}
$$

Step 4. Substituting (59), (60), (61), and (63) into (58) and equating the coefficients of $P^{i}$ to zero, we can get a series of algebraic equations. The exact solution of (56) can be gained by solving those algebraic equations.

For the (3+1)-dimensional TSF-ZK equation, we introduce the traveling wave solutions by making the following transformations

$$
\begin{aligned}
& \phi(\xi, \eta, \zeta, \tau)=\phi(E), \\
& E=\frac{k_{1} \tau^{\alpha}}{\Gamma(1+\alpha)}+\frac{k_{2} \xi^{\beta}}{\Gamma(1+\beta)}+\frac{k_{3} \eta^{\gamma}}{\Gamma(1+\gamma)}+\frac{k_{4} \zeta^{\theta}}{\Gamma(1+\theta)},
\end{aligned}
$$

where $k_{i}(i=1,2,3,4)$ are arbitrary constants. Thus, the $(3+1)$-dimensional TSF-ZK equation reduces to a nonlinear ordinary differential equation as follows:

$$
k_{1} \phi_{\xi}+a_{1} k_{2} \phi \phi_{\xi}+\left(a_{2} k_{2}^{3}+a_{3} k_{2} k_{3}^{2}+a_{4} k_{2} k_{4}^{2}\right) \phi_{\xi \xi \xi}=0 .
$$


Next, $\phi(E)$ is expanded to the following power series of the Jacobian elliptic function:

$$
\phi(E)=c_{0}+c_{1} P+\cdots+c_{N-1} P^{N-1}+c_{N} P^{N}, \quad c_{N} \neq 0,
$$

where $\phi(E)= \pm 1 / \sqrt{1 \pm e^{2 E}}$ and the function $P$ is the solution of $P_{E}(E)=P^{3}(E)-P(E)$. In (65), balancing the highest order nonlinear terms $\phi(E) \phi_{\xi}(E)$ and the highest order linear terms $\phi_{\xi \xi \xi}(E)$, we acquire $N=4$. Thus, the $\phi_{\xi \xi \xi}(E)$ can be expanded as follows:

$$
\phi(E)=c_{0}+c_{1} P+c_{2} P^{2}+c_{3} P^{3}+c_{4} P^{4}, \quad c_{4} \neq 0 .
$$

Based on (67), we obtain

$$
\begin{aligned}
\phi_{\xi}= & 4 c_{4} P^{6}+3 c_{3} P^{5}+\left(2 c_{2}-4 c_{4}\right) P^{4}+\left(c_{1}-3 c_{3}\right) P^{3} \\
& -2 c_{2} P^{2}-c_{1} P \\
\phi_{\xi \xi}= & 24 c_{4} P^{8}+15 c_{3} P^{7}+\left(8 c_{2}-40 c_{4}\right) P^{6} \\
& +\left(3 c_{1}-24 c_{3}\right) P^{5}+\left(16 c_{4}-12 c_{2}\right) P^{4} \\
& +\left(9 c_{3}-4 c_{1}\right) P^{3}+4 c_{2} P^{2}+c_{1} P, \\
\phi_{\xi \xi \xi}= & 192 c_{4} P^{10}+105 c_{3} P^{9}+\left(48 c_{2}-432 c_{4}\right) P^{8} \\
& +\left(15 c_{1}-225 c_{3}\right) P^{7}+\left(304 c_{4}-96 c_{2}\right) P^{6} \\
& +\left(147 c_{3}-27 c_{1}\right) P^{5}+\left(56 c_{2}-64 c_{4}\right) P^{4} \\
& +\left(13 c_{1}-27 c_{3}\right) P^{3}-8 c_{2} P^{2}-c_{1} P .
\end{aligned}
$$

Substituting (67) and (68) into (65) and collecting the coefficient of each power of $P^{i}(i=12,3, \cdots)$, we can present a system of algebraic equations in the following form:

$$
\begin{aligned}
P^{10} & : 4 a_{1} k_{2} c_{4}^{2}+192 c_{4}\left(a_{2} k_{2}^{3}+a_{3} k_{2} k_{3}^{2}+a_{4} k_{2} k_{4}^{2}\right)=0, \\
P^{9} & : 7 a_{1} k_{2} c_{3} c_{4}+105 c_{3}\left(a_{2} k_{2}^{3}+a_{3} k_{2} k_{3}^{2}+a_{4} k_{2} k_{4}^{2}\right)=0, \\
P^{8} & : a_{1} k_{2}\left(6 c_{2} c_{4}+3 c_{3}^{2}-4 c_{4}^{2}\right) \\
& +\left(48 c_{2}-432 c_{4}\right)\left(a_{2} k_{2}^{3}+a_{3} k_{2} k_{3}^{2}+a_{4} k_{2} k_{4}^{2}\right), \\
P^{7} & : a_{1} k_{2}\left(5 c_{1} c_{4}+4 c_{2} c_{3}-4 c_{3} c_{4}\right) \\
& +\left(15 c_{1}-225 c_{3}\right)\left(a_{2} k_{2}^{3}+a_{3} k_{2} k_{3}^{2}+a_{4} k_{2} k_{4}^{2}\right)=0, \\
P^{6} & : 4 k_{1} c_{4}+a_{1} k_{2}\left(4 c_{0} c_{4}+4 c_{1} c_{3}+2 c_{2}^{2}-6 c_{2} c_{4}+3 c_{3}^{2}\right) \\
& +\left(304 c_{4}-96 c_{2}\right)\left(a_{2} k_{2}^{3}+a_{3} k_{2} k_{3}^{2}+a_{4} k_{2} k_{4}^{2}\right)=0, \\
P^{5} & : 3 c_{3} k_{1}+a_{1} k_{2}\left(3 c_{0} c_{3}+3 c_{1} c_{2}-5 c_{1} c_{4}-5 c_{2} c_{3}\right) \\
& +\left(147 c_{3}-27 c_{1}\right)\left(a_{2} k_{2}^{3}+a_{3} k_{2} k_{3}^{2}+a_{4} k_{2} k_{4}^{2}\right)=0
\end{aligned}
$$

$$
\begin{aligned}
P^{4} & :\left(2 c_{2}-4 c_{4}\right) k_{1} \\
& +a_{1} k_{2}\left(2 c_{0} c_{2}-4 c_{0} c_{4}+c_{1}^{2}-4 c_{1} c_{3}-2 c_{2}^{2}\right) \\
& +\left(56 c_{2}-64 c_{4}\right)\left(a_{2} k_{2}^{3}+a_{3} k_{2} k_{3}^{2}+a_{4} k_{2} k_{4}^{2}\right)=0, \\
P^{3} & :\left(c_{1}-3 c_{3}\right) k_{1}+a_{1} k_{2}\left(c_{0} c_{1}-3 c_{0} c_{3}-3 c_{1} c_{2}\right) \\
& +\left(13 c_{1}-27 c_{3}\right)\left(a_{2} k_{2}^{3}+a_{3} k_{2} k_{3}^{2}+a_{4} k_{2} k_{4}^{2}\right)=0, \\
P^{2} & :-2 c_{2} k_{1}-a_{1} k_{2}\left(c_{0} c_{2}+c_{1}^{2}\right) \\
& -8 c_{2}\left(a_{2} k_{2}^{3}+a_{3} k_{2} k_{3}^{2}+a_{4} k_{2} k_{4}^{2}\right)=0, \\
P & :-c_{1} k_{1}-a_{1} k_{2} c_{0} c_{1} \\
& -c_{1}\left(a_{2} k_{2}^{3}+a_{3} k_{2} k_{3}^{2}+a_{4} k_{2} k_{4}^{2}\right)=0 .
\end{aligned}
$$

Solving the above algebraic equations, the coefficients can be written as

$$
\begin{aligned}
& c_{0}=\frac{2}{a_{1}} k\left(\sqrt[3]{12 a_{2}}-\sqrt{12 a_{2}}\right), \\
& c_{1}=c_{3}=0 \\
& c_{2}=\frac{12 k \sqrt{12 a_{2}}}{a_{1}} \\
& c_{4}=-\frac{12 k \sqrt{12 a_{2}}}{a_{1}} \\
& k_{1}=k \\
& k_{2}=k_{3}=k_{4}=-\sqrt[3]{\frac{k}{12 a_{2}}}
\end{aligned}
$$

Thus, we get the solution of $(3+1)$-dimensional TSF-ZK equation in the following form:

$$
\begin{aligned}
\phi & =\frac{2}{a_{1}} k\left(\sqrt[3]{12 a_{2}}-\sqrt{12 a_{2}}\right)+\frac{3 k \sqrt{12 a_{2}}}{a_{1}} \\
& \cdot \operatorname{sech}^{2}\left[2 \sqrt [ 3 ] { \frac { k } { 1 2 a _ { 2 } } } \left(\frac{\xi^{\beta}}{\Gamma(1+\beta)}+\frac{\eta^{\gamma}}{\Gamma(1+\gamma)}\right.\right. \\
& \left.\left.+\frac{\eta^{\theta}}{\Gamma(1+\theta)}\right)-2 k \frac{\tau^{\alpha}}{\Gamma(1+\alpha)}\right] .
\end{aligned}
$$

\section{The Property of the Dust Acoustic Waves by a Gravity Field in Collisional Dust Plasma}

According to the above section, we can obtain the exact solution of (3+1)-dimensional TSF-ZK equation. Assuming that $\alpha=\beta=\gamma=\theta$, (71) can be rewritten as

$$
\phi=\frac{2}{a_{1}} k\left(\sqrt[3]{12 a_{2}}-\sqrt{12 a_{2}}\right)+\frac{3 k \sqrt{12 a_{2}}}{a_{1}}
$$




$$
\begin{aligned}
& \cdot \operatorname{sech}^{2}\left[\frac{2}{\Gamma(1+\alpha)}\right. \\
& \left.\cdot \sqrt[3]{\frac{k}{12 a_{2}}}\left(\xi^{\beta}+\eta^{\gamma}+\eta^{\theta}-k \sqrt[3]{\frac{12 a_{2}}{k}} \tau^{\alpha}\right)\right] .
\end{aligned}
$$

Letting $\phi_{m}$ and $\omega$ be amplitude and width of solitary waves, respectively, we gain the following definitions:

$$
\begin{aligned}
\phi_{m} & =\frac{3 k \sqrt{12 a_{2}}}{a_{1}}, \\
\omega & =\frac{\Gamma(1+\alpha)}{2} \sqrt[3]{\frac{12 a_{2}}{k} .}
\end{aligned}
$$

When $\alpha=\beta=\gamma=\theta=1$, the motion velocity of the solitary wave is

$$
v=\frac{k}{\sqrt{3}} \sqrt[3]{\frac{12 a_{2}}{k}} .
$$

Furthermore, according to (17), the phase velocity of solitary waves is in the following form:

$$
v_{0}=\frac{g}{\beta_{0}} \pm \sqrt{1+\frac{T_{d}}{Z_{d}}} .
$$

6.1. Study of the Charge Properties of Dust Particle $Z d_{0}$ in Dust Acoustic Waves. According to $\phi_{m}$, we know that when $a_{1} a_{2}>$ 0 , the TSF-ZK equation has the bright soliton solution, and when $a_{1} a_{2}<0$, the $(3+1)$-dimensional TSF-ZK equation has the dark soliton solution.

Case 1. We assume that the dust particles are negatively charged; that is, $Z_{d 0}>0$. Based on the coefficient equation (19) of (3+1)-dimensional TSF-ZK equation, we know that $a_{1}$ and $a_{2}$ are real numbers and $a_{1} a_{2}<0$. Therefore, in a dust plasma system, the solution (72) of an solitary wave in the case of negative dust charge is a dark soliton. These results are consistent with those obtained by the Sagdeev potential method used by Ma [54].

Case 2. We assume that the dust particles are positively charged; that is, $Z_{d 0}<0$. Based on (19), when $T_{d}<-Z_{d 0}$, we also get that $a_{1}$ and $a_{2}$ are real numbers and $a_{1} a_{2}<0$. At this time, the solution (72) is dark soliton in the dust plasma system. When $T_{d}>-Z_{d 0}, a_{1}$ and $a_{2}$ are complex numbers; that is, solitary waves show a trend of decay, so there are no solitary waves in dust plasma system.

6.2. Study of the Parameter $k$ in Dust Acoustic Waves. Based on the extended Kudryashov method, the exact solution (72) of (3+1)-dimensional TSF-ZK equation is obtained. For the (3+1)-dimensional TSF-ZK equation and its solution, we know that the positive and negative of $k$ are the same as the positive and negative of $a_{2}$, indicating the direction of propagation of dust acoustic waves. $a_{2}>0$ represents the right traveling waves, and $a_{2}<0$ represents the left traveling waves. As long as there are dust acoustic waves in the system, where $a_{1}$ and $a_{2}$ are real numbers, there is always $a_{2}<0$. This indicates that the left traveling waves exist in the dust plasma system.

Furthermore, we know that polynomial coefficients and parameters are in the same equivalence class with the solution which is obtained by using classical method. However, according to the solution by using extended Kudryashov method, as the value of $k$ changes, the amplitude, wavelength, and velocity of the dust acoustic waves could change accordingly. From Figure 1, we find that when $k$ increases, so do the amplitude and wavelength of the solitary waves. When $k \longrightarrow \infty$, the amplitude, wavelength, and velocity of the dust acoustic waves reach their maximum values. At a physical level, dust acoustic waves are driven by electron thermal pressure and the ion pressure $T_{i}$. The increase of $k$ can be regarded as the increase of electron thermal pressure, which increases the amplitude, wavelength, and velocity of the solitary waves with small amplitude.

6.3. Study of the Fractional Order Values $\alpha, \beta, \gamma$, and $\theta$ in Dust Acoustic Waves. In this section, we study the effect of the variation of fractional order values $\alpha, \beta, \gamma$, and $\theta$ in the dust acoustic waves. Based on the soliton solution (74), we obtain Figure 2. Figure 2(a) shows that the time fractional order value $\alpha$ of differentiation has a small effect only on the position of the wave peak. It does not change the amplitude and wavelength of dust acoustic waves. As the value of $\alpha$ increases, the wave peak moves to the left. Figure 2(b) shows that the space fractional order values $\beta, \gamma$, and $\theta$ of differentiation have a small effect only on the position of the wave peak. They also do not change the amplitude and wavelength of dust acoustic waves. And as the values of $\beta, \gamma$, and $\theta$ increase, the wave peak moves to the left.

6.4. Study of the Temperature $T_{d}$ in Dust Acoustic Waves. At this point, the effect of dust temperature on dust acoustic waveforms is considered. When the dust ion temperature increases, the nonlinear term coefficient $a_{1}$ and dispersion coefficient $a_{2}$ of (3+1)-dimensional TSF-ZK equation both decrease. According to the exact solution of $(3+1)$ dimensional TSF-ZK equation, we obtain three pictures of the dust acoustic waves. Based on Figure 3, we know that the dust acoustic waveforms are constantly changing with the temperature of dust ions. Furthermore, with the increase in temperature, the motion velocity and the phase velocity of the dust acoustic waves also increase. In [55], the effect of temperature ratio between ions and electrons $\sigma=T_{i} / T_{e}$ on classical solitons is studied. The amplitude of the classical isolator increases and the characteristic width decreases with the decrease of $\sigma$, which is consistent with the waveform change of the dust acoustic waves in Figure 3.

6.5. Study of the Gravity $g$ and the Collision Frequency $\beta_{0}, \beta_{1}$ in Dust Acoustic Waves. According to the (3+1)-dimensional TSF-ZK equation and its exact solution, we can study the effect of gravity $g$ and collision frequency $\beta_{0}$ on the dust 


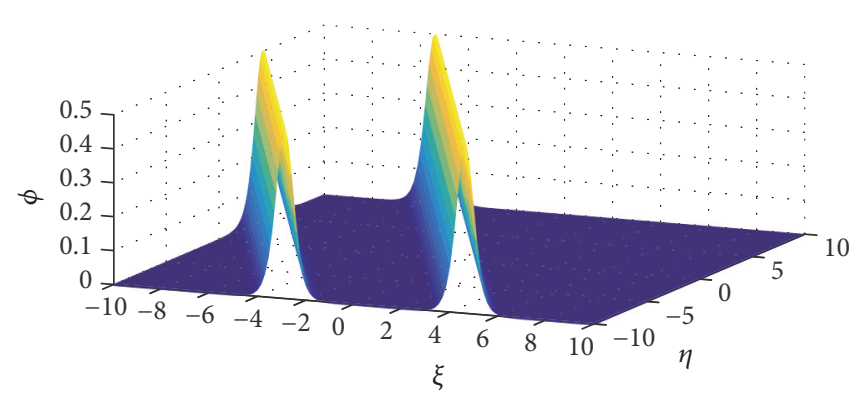

(a)

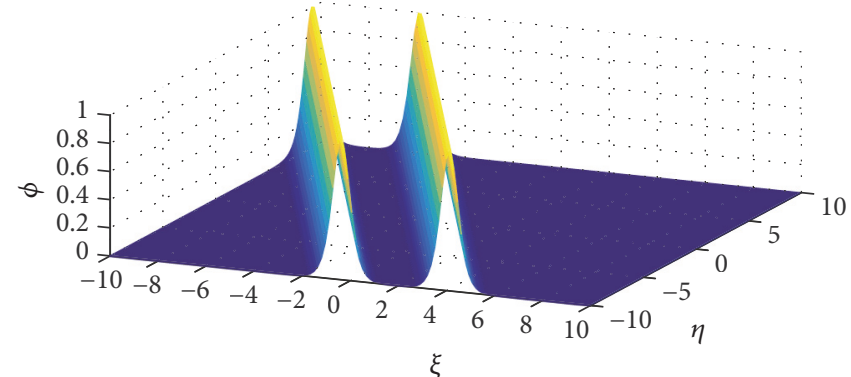

(b)

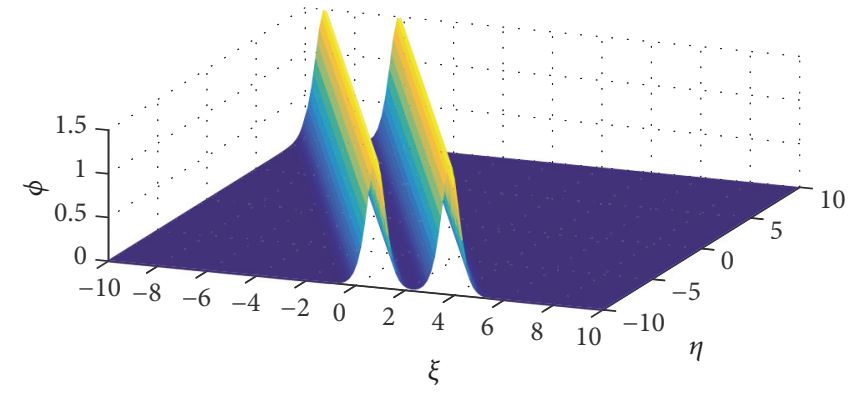

(c)

Figure 1: Plots for the evolution of the exact solution of dust acoustic solitary waves with (a) $k=0.5, t=2, a_{1}=1.67, a_{2}=a_{3}=a_{4}=1.29$, $\alpha=0.5, \beta=\gamma=\theta=1$, and $k_{2}=k_{3}=k_{4}=0.26$; (b) $k=1, t=2, a_{1}=1.67, a_{2}=a_{3}=a_{4}=1.29, \alpha=0.5, \beta=\gamma=\theta=1$, and $k_{2}=k_{3}=k_{4}=0.26$; (c) $k=1.5, t=2, a_{1}=1.67, a_{2}=a_{3}=a_{4}=1.29, \alpha=0.5, \beta=\gamma=\theta=1$, and $k_{2}=k_{3}=k_{4}=0.26$.

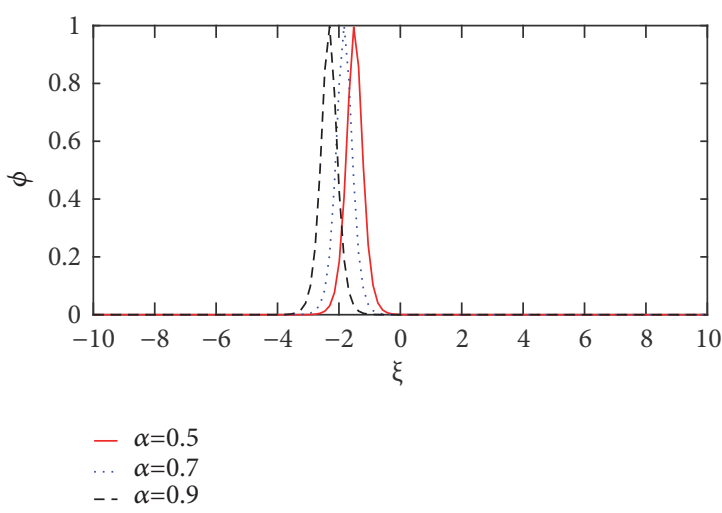

(a)

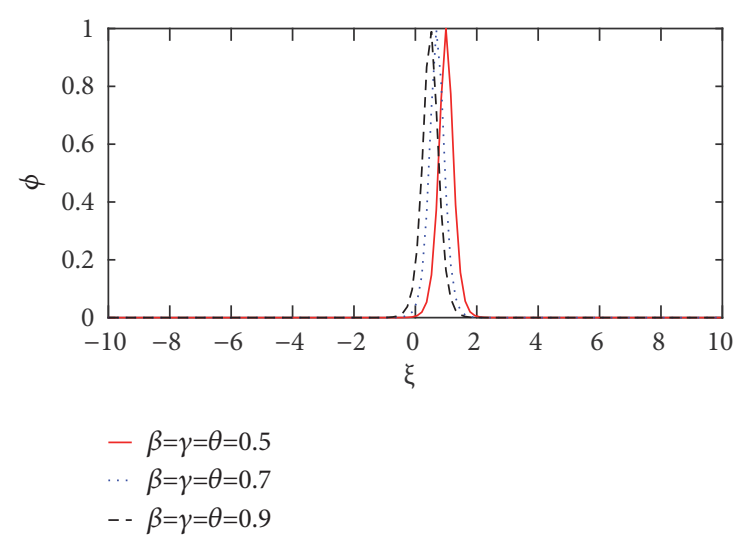

(b)

Figure 2: The dust acoustic waves with position at different values of (a) $\alpha$ for $k=2, t=2, a_{2}=a_{3}=a_{4}=1.29, \beta=\gamma=\theta=1$, and $k_{2}=k_{3}=k_{4}=0.38$; (b) $\beta, \gamma, \theta$ for $k=2, t=2, a_{2}=a_{3}=a_{4}=1.29, \alpha=0.5$, and $k_{2}=k_{3}=k_{4}=0.38$.

acoustic waves. Assuming that there are no collisions and no gravity in the neutral gas and using the same reduced perturbation method, we can get the following coefficients and phase velocity

$$
\begin{aligned}
& a_{1}=-\frac{3 v_{0}}{2}, \\
& a_{2}=\frac{1}{2 v_{0}}, \\
& v_{0}= \pm \sqrt{1+\frac{T_{d}}{Z_{d 0}}} .
\end{aligned}
$$

The result shows that, in the presence of gravity and collision frequency, that is, taking $g$ and $\beta_{0}$ into account, the phase velocity of the dust acoustic waves is increasing. Because $g>0$ and $\beta_{0}>0$, their ratio $g / \beta_{0}$ is always positive. There is no significant influence on the amplitude of the dust acoustic waves. However, using reduction perturbation analysis, we find that the collision disturbance $\beta_{1}$ of neutral gas contributes to the nonlinear term; thus the soliton solution appears in the colliding plasma. In [52], we can know that if there are unstable dust acoustic waves, that is, there is an imaginary part in the dispersion relationship, the solitary wave will not exist. The difference between our study and 


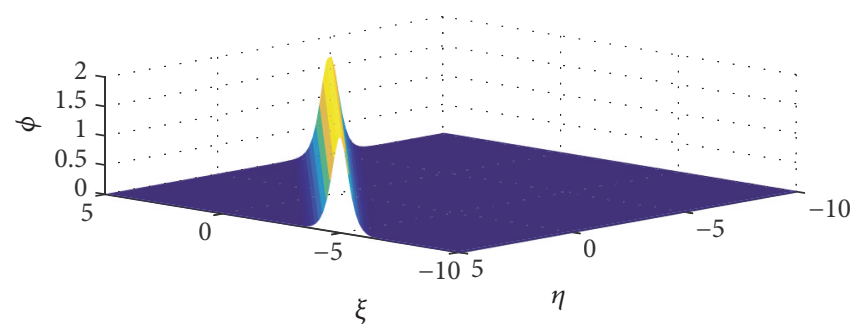

(a)

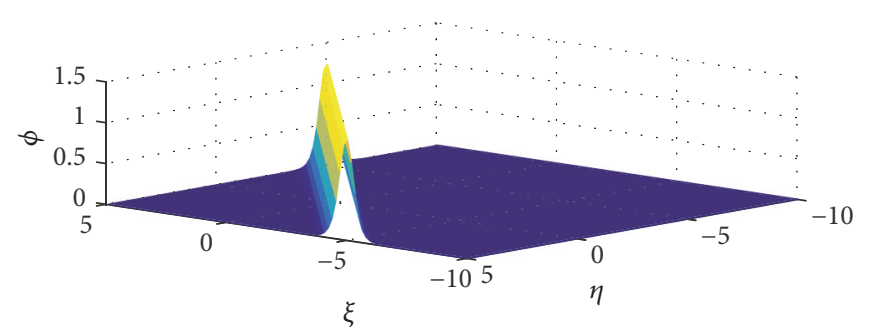

(b)

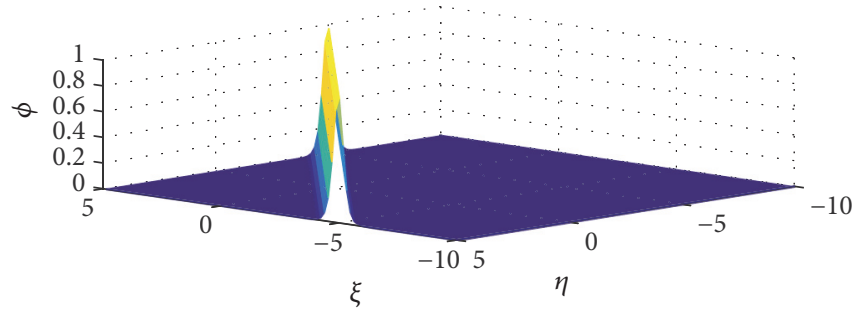

(c)

Figure 3: Plots for the evolution of the exact solution (72) of dust acoustic solitary waves with (a) $T=2, t=2, a_{1}=1.67, a_{2}=a_{3}=a_{4}=1.29$, $\alpha=0.5, \beta=\gamma=\theta=1$, and $k_{2}=k_{3}=k_{4}=0.38$; (b) $T=3, t=2, a_{1}=1.67, a_{2}=a_{3}=a_{4}=1.29, \alpha=0.5, \beta=\gamma=\theta=1$, and $k_{2}=k_{3}=k_{4}=0.38$; (c) $T=4, t=2, a_{1}=1.67, a_{2}=a_{3}=a_{4}=1.29, \alpha=0.5, \beta=\gamma=\theta=1$, and $k_{2}=k_{3}=k_{4}=0.38$.

[52] is that we consider the collision of neutral gases as the perturbation term. From the actual physical background, this view is more reasonable.

\section{Conclusion}

In this paper, according to the control equations of motion, we obtain a (3+1)-dimensional ZK equation describing the propagation of nonlinear dust acoustic waves in space. Then, we extend this equation to the fractional order for the first time and have a completely new model which is the $(3+1)$ dimensional TSF-ZK equation. The fractional equation that can describe fractal processes of the dust acoustic waves is related to the dust acoustic waves propagation with fractal properties. In the following, we study the conservation law of the $(3+1)$-dimensional TSF-ZK equation. This indicates that the dust acoustic wave described by this new model is conserved in energy during its propagation, no matter in the fractal process or in the interaction. In addition, according to the extended Kudryashov method, we get the exact solution of $(3+1)$-dimensional TSF-ZK equation. Then we draw on the exact solution to study the effect of the parameter $k$, the charge properties of dust particle $Z_{d 0}$, the fractional order values $\alpha, \beta, \gamma$, and $\theta$, the temperature $T_{d}$, the gravity $g$, and the collision frequency $\beta_{0}$ and $\beta_{1}$ on the properties of nonlinear dust acoustic waves by a gravity field in dust plasma.

\section{Data Availability}

No data is used in our research.

\section{Conflicts of Interest}

The authors declare that there are no conflicts of interest regarding the publication of this paper.

\section{Acknowledgments}

This work was supported by Nature Science Foundation of Shandong Province of China (No. ZR2018MA017) and China Postdoctoral Science Foundation funded project (No. 2017M610436).

\section{References}

[1] J. X. Ma, “Dusty plasmas," Physics, pp. 244-250, 2006.

[2] F. Li, "Dust plasma physics," Physics, vol. 9, pp. 518-521, 2006.

[3] N. N. Rao, P. K. Shukla, and M. Y. Yu, "Dust-acoustic waves in dusty plasma," Planet Space Science, vol. 38, p. 543, 1990.

[4] P. K. Shukla and V. P. Silin, "Dustion-acoustic wave," Physics Scripa, vol. 45, pp. 508-511, 1992.

[5] R. L. Merlino, A. Barkan, C. Thompson, and N. D’Angelo, "Experiments on waves and instabilities in dusty plasmas," Plasma Physics and Controlled Fusion, vol. 39, no. 5A, pp. A421A429, 1997.

[6] R. Bharuthram and P. K. Shukla, "Large amplitude ion-acoustic solitons in a dusty plasma," Planetary and Space Science, vol. 41, pp. 17-19, 1992.

[7] W.-S. Duan and Y.-R. Shi, "The effect of dust size distribution for two ion temperature dusty plasmas," Chaos, Solitons \& Fractals, vol. 18, no. 2, pp. 321-328, 2003.

[8] B. B. Kadomstev and V. I. Petviashvili, "On the stability of solitary waves in weakly dispersive media," Doklady Akademii nauk SSSR, vol. 15, pp. 539-541, 1970.

[9] S. Singh and T. Honzawa, "Kadomtsev-Petviashivili equation for an ion-acoustic soliton in a collisionless weakly relativistic plasma with finite ion temperature," Physics Fluids B: Plasma Physics, vol. 5, no. 7, pp. 2093-2097, 1993.

[10] M.-M. Lin and W.-S. Duan, " $(2+1)$ dimensional nonlinear waves in a two-ion-temperature warm dusty plasma 
considering the dust size distribution," Chaos, Solitons \& Fractals, vol. 21, no. 2, pp. 325-333, 2004.

[11] W.-S. Duan, "The Kadomtsev-Petviashvili (KP) equation of dust acoustic waves for hot dust plasmas," Chaos, Solitons \& Fractals, vol. 14, no. 3, pp. 503-506, 2002.

[12] T. S. Gill, N. S. Saini, and H. Kaur, “The Kadomstev-Petviashvili equation in dusty plasma with variable dust charge and two temperature ions," Chaos, Solitons \& Fractals, vol. 28, no. 4, pp. 1106-1111, 2006.

[13] H. W. Yang, Z. H. Xu, D. Z. Yang, X. R. Feng, B. S. Yin, and H. H. Dong, "ZK-Burgers equation for three-dimensional Rossby solitary waves and its solutions as well as chirp effect," Advances in Difference Equations, vol. 2016, no. 167, 2016.

[14] M. Guo, Y. Zhang, M. Wang, Y. Chen, and H. Yang, "A new ZKILW equation for algebraic gravity solitary waves in finite depth stratified atmosphere and the research of squall lines formation mechanism," Computers \& Mathematics with Applications, vol. 75, no. 10, pp. 3589-3603, 2018.

[15] L. Fu, Y. D. Chen, and H. W. Yang, "Time-space fractional coupled generalized Zakharov-Kuznetsov equations set for rossby solitary waves in two-layer fluids," Mathematics, vol. 7, no. 1, p. 41, 2019.

[16] M. Han, L. Zhang, Y. Wang, and C. M. Khalique, "The effects of the singular lines on the traveling wave solutions of modified dispersive water wave equations," Nonlinear Analysis: Real World Applications, vol. 47, pp. 236-250, 2019.

[17] J. T. Ha, H. Q. Zhang, and Q. L. Zhao, "Exact solutions for a Dirac-type equation with N-fold Darboux transformation," Journal of Applied Analysis and Computation, vol. 9, pp. 200210, 2019.

[18] M. Liu, X. Li, and Q. Zhao, "Exact solutions to Euler equation and Navier-Stokes equation," Zeitschrift für Angewandte Mathematik und Physik, vol. 70, no. 2, pp. 1-13, 2019.

[19] H. K. Qi, L. D. Liu, and X. Z. Meng, "Dynamics of a nonautonomous stochastic sis epidemic model with double epidemic hypothesis," Complexity, vol. 2017, Article ID 4861391, 14 pages, 2017.

[20] F. X. Song and H. W. Yang, "Modeling and analysis of fractional neutral disturbance waves in arterial vessels," Mathematical Modelling of Natural Phenomena, vol. 14, no. 3, p. 301, 2019.

[21] C. N. Lu, L. Y. Xie, and H. W. Yang, "Analysis of Lie symmetries with conservation laws and solutions for the generalized $(3+1)$-dimensional time fractional Camassa-Holm-KadomtsevPetviashvili equation," Computers \& Mathematics with Applications, vol. 77, no. 12, pp. 3154-3171, 2019.

[22] Z. Z. Yue and Y. M. Zou, "New uniqueness results for fractional differential equation with dependence on the first order derivative," Advances in Difference Equations, vol. 2019, no. 1, p. 38, 2019.

[23] H. Yang, J. Sun, and C. Fu, "Time-fractional Benjamin-Ono equation for algebraic gravity solitary waves in baroclinic atmosphere and exact multi-soliton solution as well as interaction," Communications in Nonlinear Science and Numerical Simulation, vol. 71, pp. 187-201, 2019.

[24] S. M. Meng and Y. J. Cui, "Multiplicity results to a conformable fractional differential equations involving integral boundary condition," Complexity, vol. 2019, Article ID 8402347, 8 pages, 2019.

[25] Y. L. Li, F. W. Liu, I. W. Turner, and T. Li, "Time-fractional diffusion equation for signal smoothing," Applied Mathematics and Computation, vol. 326, pp. 108-116, 2018.
[26] S. Aman, Q. Al-Mdallal, and I. Khan, "Heat transfer and second order slip effect on MHD flow of fractional Maxwell fluid in a porous medium," Journal of King Saud University-Science, 2018.

[27] Q. M. Al-Mdallal and A. S. Abu Omer, "Fractional-order Legendre-collocation method for solving fractional initial value problems," Applied Mathematics and Computation, vol. 321, pp. 74-84, 2018.

[28] Q. Song and Z. Bai, "Positive solutions of fractional differential equations involving the Riemann-Stieltjes integral boundary condition," Advances in Difference Equations, vol. 2018, Article ID 183, 2018.

[29] Y. W. Ren, M. S. Tao, H. D. Dong, and H. W. Yang, "Analytical research of $(3+1)$-dimensional Rossby waves with dissipation effect in cylindrical coordinate based on Lie symmetry approach," Advances in Difference Equations, vol. 2019, p. 13, 2019.

[30] C. Lu, C. Fu, and H. Yang, "Time-fractional generalized Boussinesq equation for Rossby solitary waves with dissipation effect in stratified fluid and conservation laws as well as exact solutions," Applied Matheamtics and Computation, vol. 327, pp. 104-116, 2018.

[31] C. Fu, C. N. Lu, and H. W. Yang, "Time-space fractional $(2+1)$ dimensional nonlinear Schrodinger equation for envelope gravity waves in baroclinic atmosphere and conservation laws as well as exact solutions," Advances in Difference Equations, vol. 2018, p. 20, 2018.

[32] X. Meng, F. Li, and S. Gao, "Global analysis and numerical simulations of a novel stochastic eco-epidemiological model with time delay," Applied Mathematics and Computation, vol. 339, pp. 701-726, 2018.

[33] Y. Tian, Y. Wei, and S. Sun, "Multiplicity for fractional differential equations with $p$-Laplacian," Boundary Value Problems, vol. 2018, p. 127, 2018.

[34] B. Kilic and M. Inc, "The First Integral Method for the time fractional Kaup-Boussinesq System with time dependent coefficient," Applied Mathematics and Computation, vol. 254, pp. 70-74, 2015.

[35] B. Lu, "The first integral method for some time fractional differential equations," Journal of Mathematical Analysis and Applications, vol. 395, no. 2, pp. 684-693, 2012.

[36] N. Shang and B. Zheng, "Exact solutions for three fractional partial differential equations by the $\left(\mathrm{G}^{\prime} / \mathrm{G}\right)$ method," International Journal of Applied Mathematics, vol. 43, no. 3, pp. 1-6, 2013.

[37] B. Zheng, " $\left(G^{\prime} / G\right)$-expansion method for solving fractional partial differential equations in the theory of mathematical physics," Communications in Theoretical Physics, vol. 58, no. 5, pp. 623-630, 2012.

[38] Y. Zhou and W. X. Ma, "Complexiton solutions to soliton equations by the Hirota method," Journal of Mathematical Physics, vol. 58, no. 10, Article ID 101511, 2017.

[39] W.-X. Ma and Y. Zhou, "Lump solutions to nonlinear partial differential equations via Hirota bilinear forms," Journal of Differential Equations, vol. 264, no. 4, pp. 2633-2659, 2018.

[40] Y. Gurefe, E. Misirli, A. Sonmezoglu, and M. Ekici, "Extended trial equation method to generalized nonlinear partial differential equations," Applied Mathematics and Computation, vol. 219, no. 10, pp. 5253-5260, 2013.

[41] S. M. Guo, L. Q. Mei, Y. Li, and Y. F. Sun, "The improved fractional sub-equation method and its applications to the space-time fractional differential equations in fluid mechanics," Physics Letters A, vol. 376, no. 4, pp. 407-411, 2012. 
[42] R. Zhang, L. Yang, J. Song, and H. Yang, “(2+1) dimensional Rossby waves with complete Coriolis force and its solution by homotopy perturbation method," Computers \& Mathematics with Applications, vol. 73, no. 9, pp. 1996-2003, 2017.

[43] M. Tao, N. Zhang, D. Gao, and H. Yang, "Symmetry analysis for three-dimensional dissipation Rossby waves," Advances in Difference Equations, vol. 2018, article 300, 2018.

[44] A. Biswas, "1-Soliton solution of the generalized ZakharovKuznetsov equation with nonlinear dispersion and timedependent coefficients," Physics Letters A, vol. 373, no. 33, pp. 2931-2934, 2009.

[45] M. Guo, H. Dong, J. Liu, and H. Yang, "The time-fractional mZK equation for gravity solitary waves and solutions using sech-tanh and radial basis function method," Nonlinear Analysis: Modelling and Control, vol. 24, no. 1, pp. 1-19, 2019.

[46] C. M. Khalique and G. Magalakwe, "Combined sinh-coshGordon equation: Symmetry reductions, exact solutions and conservation laws," Quaestiones Mathematicae, vol. 37, no. 2, pp. 199-214, 2014.

[47] E. Misirli and S. M. Ege, "A new method for solving nonlinear fractional differential equations," New Trends in Mathematical Science, vol. 1, no. 5, pp. 225-233, 2017.

[48] B. J. Zhao, R. Y. Wang, W. J. Sun, and H. W. Yang, "Combined ZK-mZK equation for Rossby solitary waves with complete Coriolis force and its conservation laws as well as exact solutions," Advances in Difference Equations, vol. 2018, no. 1, p. 42, 2018.

[49] M. Guo, C. Fu, Y. Zhang, J. X. Liu, and H. W. Yang, "Study of ionacoustic solitary waves in a magnetized plasma using the threedimensional time-space fractional Schamel-KdV equation," Complexity, vol. 2018, Article ID 6852548, 17 pages, 2018.

[50] S. A. El-Wakil and E. M. Abulwafa, "Formulation and solution of space-time fractional Boussinesq equation," Nonlinear Dynamics, vol. 80, no. 1, pp. 167-175, 2015.

[51] W.-S. Duan, " $3+1$ dimensional envelop waves and its stability in magnetized dusty plasma," Chaos, Solitons \& Fractals, vol. 27, no. 4, pp. 926-929, 2006.

[52] N. D’Angelo, "Dust acoustic waves excited by gravity in a collisional plasma," Physics Letters A, vol. 304, no. 3-4, pp. 102$105,2002$.

[53] Q. Zhao and S.-K. Liu, "Application of Jacobi elliptic functions in the atmospheric and oceanic dynamics: Studies on twodimensional nonlinear Rossby waves," Chinese Journal of Geophysics, vol. 49, no. 4, pp. 965-970, 2006.

[54] J. X. Ma and J. Liu, "Dust-acoustic soliton in a dusty plasma," Physics of Plasmas, vol. 4, no. 2, pp. 253-255, 1997.

[55] J. Y. Wang, X. P. Cheng, Y. Zeng, Y. X. Zhang, and N. Y. Ge, "Quasi-soliton solution of Korteweg-de Vries equation and its application in ion acoustic waves," Acta Physica Sinica, vol. 67, Article ID 110201, 2018. 


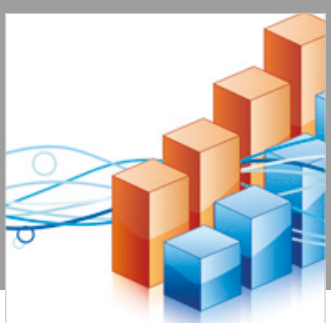

Advances in

Operations Research

\section{-n-m}
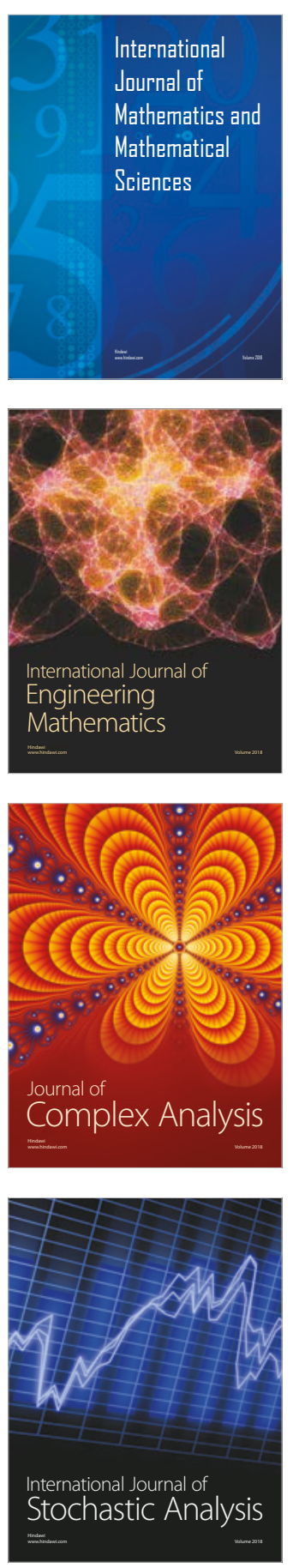
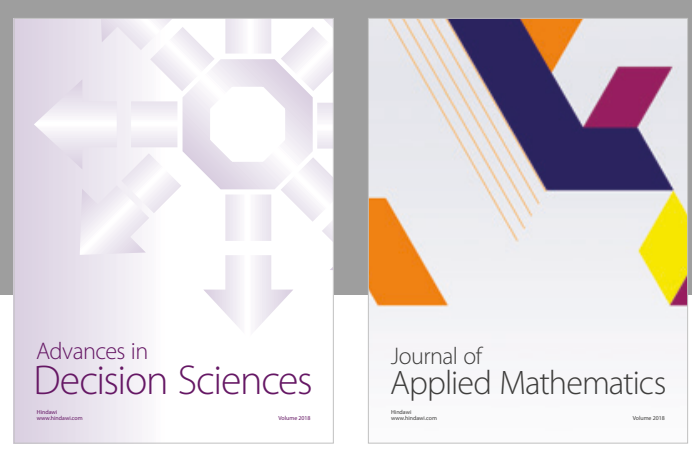

Journal of

Applied Mathematics
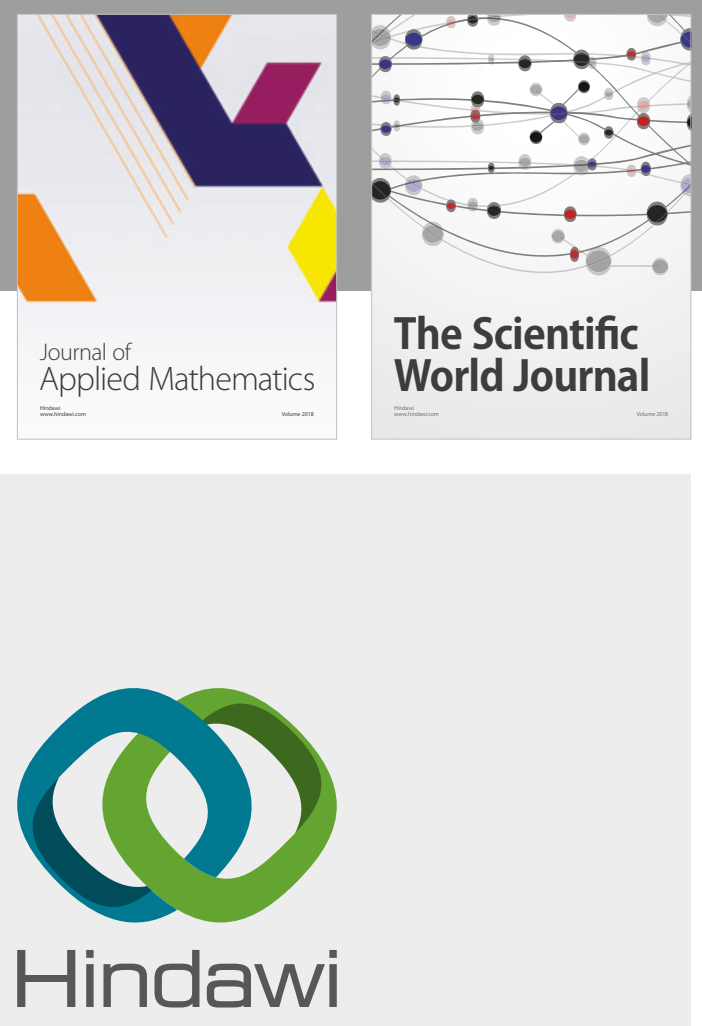

Submit your manuscripts at

www.hindawi.com

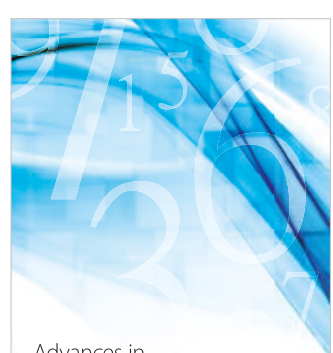

Advances in
Numerical Analysis
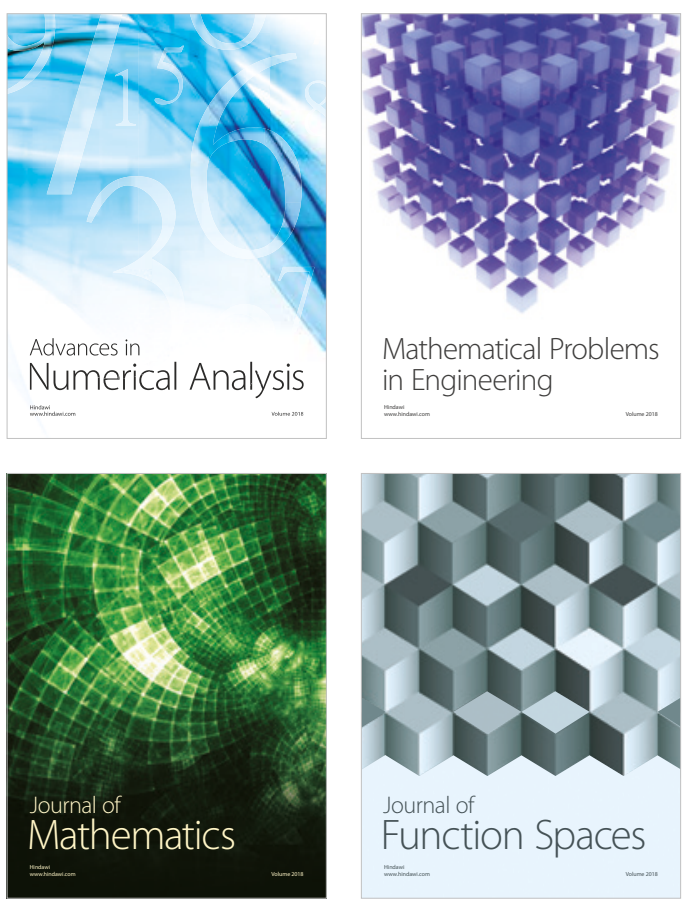

Mathematical Problems in Engineering

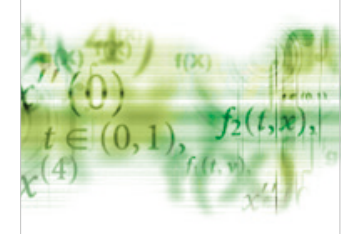

International Journal of

Differential Equations

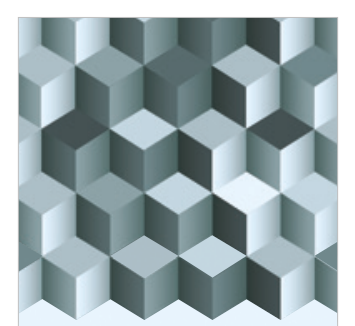

Journal of

Function Spaces
The Scientific

World Journal

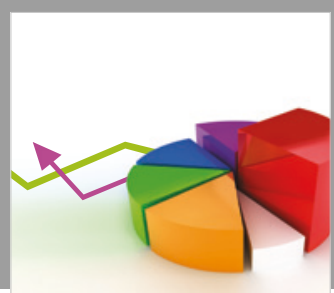

Journal of

Probability and Statistics
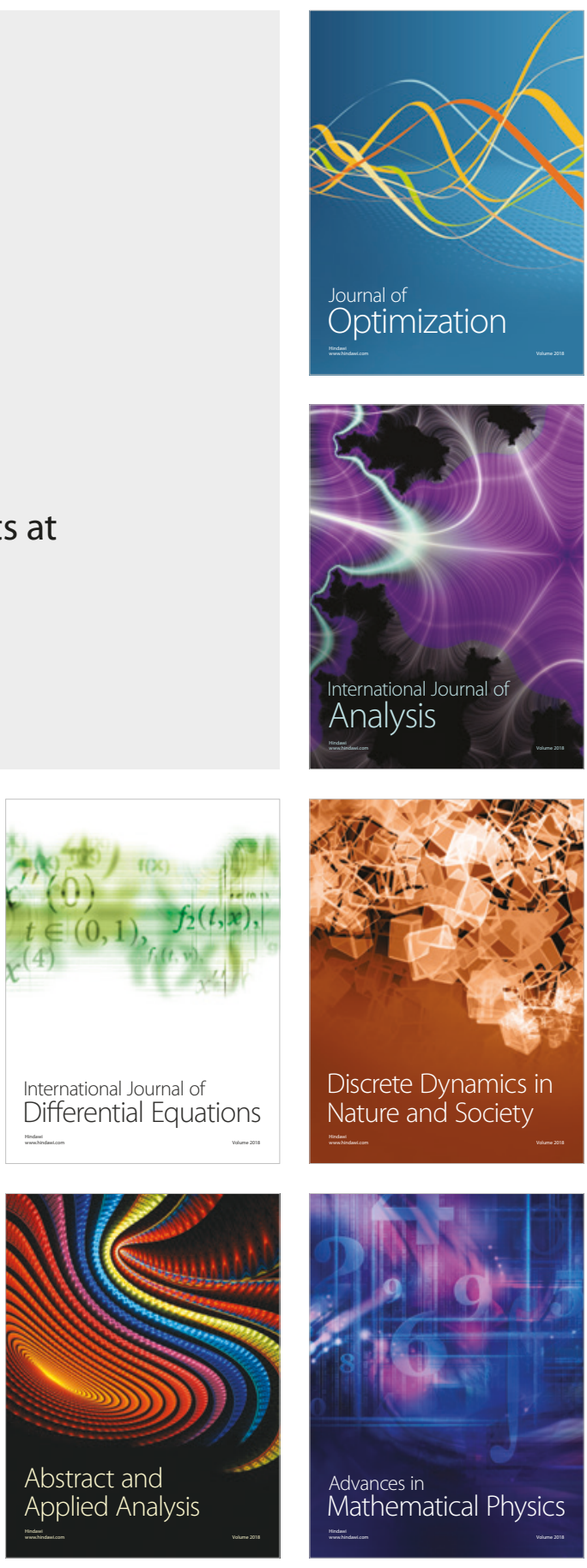